\title{
Filtered sub-grid constitutive models for fluidized gas-particle flows constructed from 3-D simulations
}

\author{
Avik Sarkar, ${ }^{\mathrm{a}, \mathrm{b}, *}$, Fernando E. Milioli ${ }^{\mathrm{c}}$, Shailesh Ozarkar ${ }^{\mathrm{d}}$, Tingwen Li ${ }^{\mathrm{e}, \mathrm{f}}$, \\ Xin Sun ${ }^{\mathrm{a}}$, Sankaran Sundaresan ${ }^{\mathrm{c}}$ \\ a Fundamental and Computational Sciences Directorate, Pacific Northwest National Laboratory, PO Box 999, \\ MSIN: K7-90, Richland, WA 99352, United States \\ b Current address: Worldwide Research \& Development, Pfizer Inc., MS 8156-014, Eastern Point Road, Groton, \\ CT 06340, United States \\ c Chemical and Biological Engineering, Princeton University, A-315 Engineering Quadrangle, Princeton \\ University, Princeton, NJ 08544, United States \\ d ANSYS Inc, Canonsburg, PA 15317, United States \\ e National Energy Technology Laboratory, 3610 Collins Ferry Rd. Morgantown, WV 26507, United States \\ f AECOM, 3610 Collins Ferry Rd. Morgantown, WV 26507, United States \\ *Corresponding author. Email: aviksarkar2@gmail.com, avik.sarkar@pfizer.com. Phone: +1 (860) 441-6533.
}

\begin{abstract}
The accuracy of fluidized-bed CFD predictions using the two-fluid model can be improved significantly, even when using coarse grids, by replacing the microscopic kinetic-theory-based closures with coarsegrained constitutive models. These coarse-grained constitutive relationships, called filtered models, account for the unresolved gas-particle structures (clusters and bubbles) via sub-grid corrections. Following the previous 2-D approaches of Igci et al. [AIChE J., 54(6), 1431-1448, 2008] and Milioli et al. [AIChE J., 59(9), 3265-3275, 2013], new closures for the filtered inter-phase drag and stresses in the gas and particle phases are constructed from highly-resolved 3-D simulations of gas-particle flows. These new closure relations are then validated through the bubbling-fluidized-bed challenge problem presented by National Energy Technology Laboratory and Particulate Solids Research Inc.
\end{abstract}

Keywords: Computational fluid dynamics (CFD); Constitutive models; Fluidization; Multiphase flow; Scale-up; Two-fluid model. 


\section{Introduction}

Gas-particle flows in fluidized and fast-fluidized beds manifest persistent fluctuations in particle volume fraction and phase velocities that span a wide range of length and time scales. These fluctuations are associated with clusters and streamers of particles in dilute flows and bubble-like voids at higher particle volume fractions. Although the formation of such structures, which can be as small as a few particle diameters, is predicted by two-fluid models supplemented with simple phenomenological relationships (Glasser et al., 1996, 1997; Glasser et al., 1998) or more elaborate kinetic theory-based closures for particle phase stress (Ding and Gidaspow, 1990; Gidaspow, 1994), resolving them explicitly using a sufficiently fine grid is impractical: $O\left(10^{6}\right)$ cells would be required for two-dimensional (2-D) simulations and $O\left(10^{9}\right)$ cells for three-dimensional (3-D) simulations of commercial-scale devices, which are well beyond current computational capabilities (Sundaresan, 2000).

Filtered two-fluid models suitable for coarse-grid simulations seek to account for the effects of finescale (unresolved) particle clusters and gas bubbles on the resolved flow via coarse-grained closure models. This approach is similar to large-eddy simulations of single-phase turbulent flows (Pope, 2000). As discussed below, several research groups have sought to formulate coarse closures on the basis of statistics generated by systematically filtering results from highly resolved simulations of flows in model geometries. Our research group has previously published constitutive models deduced from highly resolved flows simulations of fluidization in 2-D periodic domains (e.g., see Igci and Sundaresan (2011a), and Milioli et al. (2013)).

In the present study, highly resolved simulations of gas-particle flows have been performed in 3-D periodic domains (Fig. 1), and analyzed to construct new 3-D filtered models for gas-particle drag, as well as stresses in the gas and particle phases. Comparison of these models with previously developed 2-D corrections exposes the similarities and differences. The present study also proposes a new model for the solids stress anisotropy. The influence of solids-phase material properties on the closures is briefly explored by performing a few additional simulations with smaller and denser particles. Coarse-grid 
simulations of the National Energy Technology Laboratory (NETL)/ Particulate Solids Research Inc. (PSRI) bubbling fluidized bed challenge problem (Shadle et al., 2011) are then performed to assess the predictions afforded by the filtered closure relations proposed in this study.
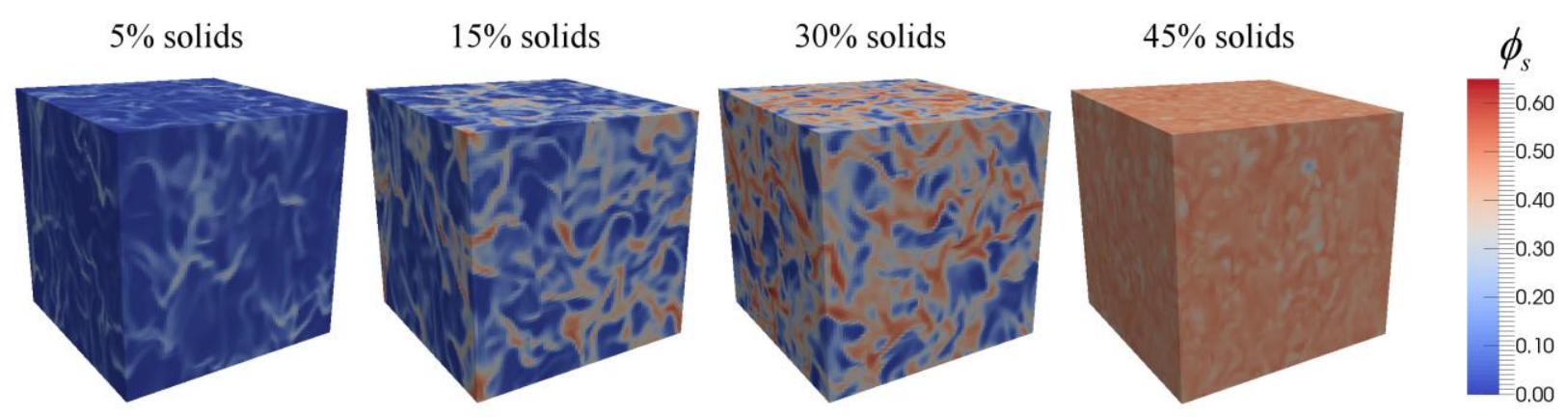

Fig. 1. Snapshots of particle volume fraction fields obtained in highly resolved, 3-D simulations of gasfluidization in periodic domains for different domain-averaged solids loading (5, 15, 30 and 45 vol\%). The color corresponds to local solid fraction $\phi_{s}$. Results from such highly resolved simulations are analyzed to construct coarse-grained constitutive relations for filtered two-fluid models.

\section{Background}

It is straightforward to coarse-grain the two-fluid model and obtain the general form of the filtered two-fluid model (Fox, 2014; Igci et al., 2008; O'Brien, 2014). One is then left with the task of developing coarse-grained closure relations for the fluid-particle interaction force and the effective stresses in the gas and particle phases. From a budget analysis, Parmentier et al. (2012) identified the correction to the gasparticle drag to be the most significant, with the effective stresses playing a lesser role. Indeed, modified drag coefficients that account for the effects of clusters have been proposed much earlier in the literature (Li and Kwauk, 1994), while deducing coarse-grained closures through systematic filtering of highly resolved simulation results is relatively more recent (Andrews et al., 2005; Igci et al., 2008; Igci and Sundaresan, 2011a; Milioli et al., 2013). The highly resolved simulations can be grouped into different categories: fluidization in (i) periodic domains (Andrews et al., 2005; Igci et al., 2008; Igci and 
Sundaresan, 2011a; Milioli et al., 2013), (ii) small beds including gas entrance, bounding walls, and gas exit (Igci and Sundaresan, 2011b; Parmentier et al., 2012), and (iii) periodic segment of a riser (Özel et al., 2013). Most studies focused on 2-D simulations, while few have considered 3-D systems. Although some quantitative differences are found in the closures obtained through these different test problems, the general characteristics of coarse closures remain very similar. More recently, Sarkar et al. (2013, 2014b) extended this method and developed the filtered drag models for fluidized beds with immersed cooling tube arrays.

Not surprisingly, the filter size $\Delta_{\text {filter }}$ (see Fig. 2) is a key parameter in the coarse-grained closures. Additionally, the filtered particle volume fraction, $\bar{\phi}_{s}$, emerges as an important marker characterizing the sub-filter scale structures in every model proposed thus far. Models involving only $\bar{\phi}_{s}$ to characterize the sub-filter scale (Igci et al., 2008; Igci and Sundaresan, 2011a; Parmentier et al., 2012; Ozel et al., 2013; Sarkar et al., 2013) are henceforth referred to as one-marker models. It should be noted that the model by Parmentier et al. (2012) includes a dynamic correction, which could be viewed as an implicit second marker. Although verification (Igci and Sundaresan, 2011b; Sarkar et al., 2014b) and validation (Igci et al., 2012) studies with the one-marker drag model generally show improvements in predictions, quantitative discrepancies remain, motivating researchers to seek more detailed filtered closures.

Recent work by Milioli et al. (2013) analyzed results from highly resolved 2-D simulations and found that the coarse-grained drag coefficient depends appreciably on filtered gas-particle slip velocity, $\tilde{v}_{\text {slip }}$, in addition to $\Delta_{\text {filter }}$ and $\bar{\phi}_{s}$. Analysis of results from 3-D simulations of a dense fluidized bed by Schneiderbauer and Pirker (2014) confirms this dependence. The coarse-grained drag coefficient closures in these studies are labeled as two-marker models. These studies did not observe any definitive dependence of the filtered stresses on the second marker $\tilde{v}_{\text {slip }}$.

These findings prompted the present study, where we have performed simulations of gas-fluidization in 3-D domains and probed the effect of $\tilde{v}_{\text {slip }}$ on the coarse-grained drag coefficient in order to bring forth 
the similarities and differences between 2-D and 3-D analyses. We also advance a model for stress anisotropy, which is well-known in granular flows (Campbell, 1997; Natarajan et al., 1995).

\section{Highly-resolved 3-D simulations and filtering procedure}

Details of the kinetic-theory-based, two-fluid model equations used to describe the gas-particle flow (Agrawal et al., 2001; Ding and Gidaspow, 1990; Gidaspow, 1994) are not presented here; for a summary of the microscopic equations used in the highly resolved simulations, please refer to Agrawal et al. (2001) and Sarkar et al. (2013). The gas-particle drag is calculated using the Wen and Yu (1966) drag model. As in Sarkar et al. (2013, 2014), the simplified algebraic approximation of the granular energy equation is used. The periodic-domain simulations were performed using the open-source code MFiX (Multiphase Flow with Interphase eXchanges) from NETL.

Following earlier approaches (Igci et al., 2008; Igci and Sundaresan, 2011a), highly-resolved simulations of fluidization of particles by a gas in 3-D periodic domains have been performed in the present study (Fig. 1). The domain-average particle volume fractions simulated range from 0.01 to 0.60 , spanning dilute to dense flow regimes (see Table 1). The pressure drop across the vertical periodic boundaries, i.e., along Y-axis, is specified such that the suspension weight is balanced. After initial transients, the flows achieve statistical steady state, i.e., the gas and solids velocities Favre-averaged over the entire domain (refer to Eqs. 4 and 5) attain steady levels but instantaneously fluctuate around a mean value (Agrawal et al., 2001; Andrews et al., 2005).

Although the results will be presented in dimensionless form, it is instructive to consider a typical gas-particle system representing fluidization of a solid sorbent being assessed for post-combustion $\mathrm{CO}_{2}$ capture (Miller et al., 2014; Sarkar et al., 2014a), which motivated the present study. Highly resolved simulations are performed in a periodic unit cell of size $13.50 \times 13.50 \times 13.50$ dimensionless units, lengths being scaled using $v_{t}^{2} / g ; v_{t}$ and $g$ being the terminal settling velocity of the particle and 
acceleration due to gravity, respectively. For the carbon-capture particles described in Table 1, this corresponds to physical dimension of $0.10 \mathrm{~m} \times 0.10 \mathrm{~m} \times 0.10 \mathrm{~m}$. In order to resolve the fine-scale particle clusters, a grid resolution of $\Delta_{\text {grid }}=8.33 d_{p}$ was used $(500,000+$ cells for each simulation). At this resolution, filtered measurements are essentially grid-size independent (Sarkar et al., 2013). This grid size is finer than that used by Igci and Sundaresan (2011a) in their 2-D and 3-D studies $\left(\Delta_{\text {grid }}=16.67 d_{p}\right)$ and by Milioli et al. (2013) in their 2-D simulations ( $\Delta_{\text {grid }} \geq 16.67 d_{p}$ ). The characteristic scales used to render the results dimensionless are same as those are utilized in previous studies (Agrawal et al., 2001; Igci et al., 2008; Igci and Sundaresan, 2011a; Milioli et al., 2013), and are summarized in Table 1, where dimensionless quantities are marked by an asterisk $(*)$.

A large number of snapshots of flow at various times in statistical steady state are recorded; from each snapshot, filtered statistics are gathered for filtering windows with different edge lengths (i.e. filter size $\Delta_{\text {filter }}$, see Fig. 2) centered at various points in the periodic domain.

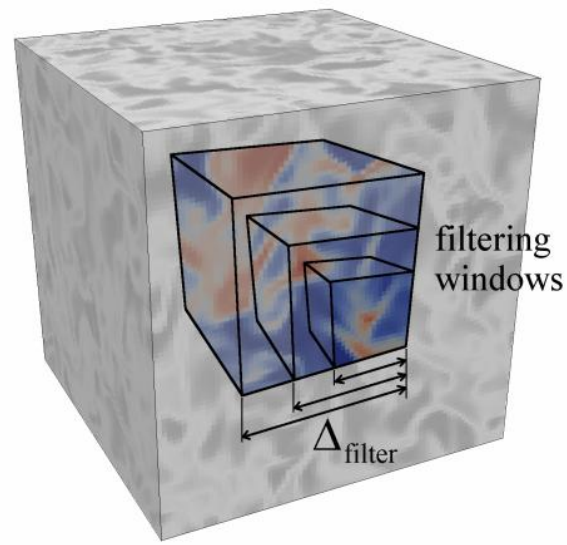

Fig. 2. Schematic of the filtering procedure, showing filtering windows of different sizes $\Delta_{\text {filter }}$ constructed within the periodic domain. For each snapshot, windows of varying sizes and at various locations are constructed to gather filtered statistics. 
For each filtering window, a uniform 'box' filter $G(x, y, z)$ is defined such that:

$$
\iiint_{\substack{\text { filtering } \\ \text { window }}} G(x, y, z) d x d y d z=1
$$

which yields $G(x, y, z)=1 / \Delta_{\text {filter }}^{3}$. Using this box filter, the filtered flow variables are defined as:

$$
\begin{aligned}
& \bar{\phi}_{s}=\iiint_{\substack{\text { filtering } \\
\text { window }}} \phi_{s} G(x, y, z) d x d y d z, \\
& \bar{\phi}_{g}=1-\bar{\phi}_{s}=\iiint_{\substack{\text { filtering } \\
\text { window }}} \phi_{g} G(x, y, z) d x d y d z, \\
& \bar{\phi} \tilde{\boldsymbol{v}}_{s}=\iiint_{\substack{\text { filtering } \\
\text { window }}} \phi_{s} \boldsymbol{v}_{s} G(x, y, z) d x d y d z, \\
& \bar{\phi}_{g} \tilde{\boldsymbol{v}}_{g}=\iiint_{\substack{\text { filtering } \\
\text { window }}} \phi_{g} \boldsymbol{v}_{g} G(x, y, z) d x d y d z,
\end{aligned}
$$

where $\bar{\phi}_{i}$ is the filtered volume fraction and $\tilde{\boldsymbol{v}}_{i}$ is the filtered velocity of phase ' $i$ ' $(i=s, g)$. The overbars denote simple volume-averaged filtered quantities (e.g., Eqs. 2 and 3) and the overhanging tildes represent phase-fraction-weighted filtered velocities (i.e., Favre averaging, Eqs. 4 and 5).

Applying the filtering operation on the microscopic mass and momentum conservation equations yields the filtered conservation equations (Eqs. 6-12 in Table 2; derivation presented in Igci et al., 2008, and Igci, 2011). The filtered conservation equations are similar in form to their unfiltered counterparts, but contain several residual correlations requiring closure relations; here, the prime denotes the random fluctuations around the filtered value of the variable; for example, $\phi_{s}^{\prime}=\phi_{s}-\bar{\phi}_{s}$ and $v_{s}^{\prime}=v_{s}-\tilde{v}_{s}$.

It has been shown in previous studies (Agrawal et al., 2001, Andrews et al., 2005, and Igci et al., 2008) that the contribution to the filtered pressure and viscosity of the particle phase arising through the residual correlation $\rho_{s} \overline{\phi_{s} v_{s}^{\prime} v_{s}^{\prime}}$ is much larger than those due to the filtered kinetic-theory pressure $\left(\bar{p}_{k i n, s}\right)$ and filtered solids viscosity $\left(\bar{\mu}_{s}\right)$, respectively, for filter sizes of practical interest in coarse-grid 
simulations (typically larger than $50 d_{p}$ ). With this in mind, the present analysis does not seek closures for the filtered granular energy balance.

\section{One-marker filtered gas-solid drag}

Following Igci et al. (2008), the term, $-\overline{\phi_{s}^{\prime} \nabla \cdot \sigma_{g}^{\prime}}$ in the filtered momentum balance is absorbed in the filtered gas-solid drag as: $\overline{\boldsymbol{F}}_{g s}=\overline{\boldsymbol{f}}_{g s}-\overline{\phi_{s}^{\prime} \nabla \cdot \sigma_{g}^{\prime}}$ (Eq. 13). Its contribution to $\overline{\boldsymbol{F}}_{g s}$ is discussed in Sec. 5.

Constitutive models for the gas-solid drag are expressed as corrections $\left(1-H_{3 \mathrm{D}}\right.$, see Eq. 13) to the microscopic drag coefficient $\bar{\beta}_{g s, \text { micro }}$ (presently given by the Wen and Yu, 1966, model but calculated using $\bar{\phi}_{s}, \tilde{v}_{s}$, and $\tilde{v}_{g}$ instead of the microscopic values). Figure 3 a presents the one-marker form of the filtered drag correction $H_{3 \mathrm{D}}^{\text {one }}$ computed from 3-D simulations.

The new 3-D one-marker drag results are qualitatively similar to the 2-D and 3-D models presented in Igci and Sundaresan (2011a), but with notable quantitative differences. The filtered correction $H_{3 \mathrm{D}}^{\text {one }}$ is mostly smaller than the $h_{2 \mathrm{D}}$ values given by Igci and Sundaresan (2011a) (solid line in Fig. 3a). This suggests that the gas-solid suspension is relatively less heterogeneous in 3-D simulations compared to 2D idealizations, especially for $\bar{\phi}_{s}>0.45$. The presence of an additional dimension in 3-D allows more pathways for the gas to bypass clusters, thus suppressing the more drastic channeling/segregation behavior predicted by 2-D simulations. The correlation for $h_{3 \mathrm{D}}$ presented by Igci and Sundaresan (2011a) does not account for the contribution of $-\overline{\phi_{s}^{\prime} \nabla \cdot \sigma_{g}^{\prime}}$. This omission, however, does not jeopardize the previous conclusions since the removal of $-\overline{\phi_{s}^{\prime} \nabla \cdot \sigma_{g}^{\prime}}$ actually produces only very small variations on $H_{3 \mathrm{D}}^{\text {one }}$, as shown in Fig. $3 \mathrm{~b}$.

Quantitative differences between $H_{3 \mathrm{D}}^{\text {one }}$ in the present study and $h_{3 \mathrm{D}}$ given by Igci and Sundaresan (2011a) (dotted line in Fig. 3a) are attributed to improved grid resolution, larger simulation domains and a 
greater number of simulations at different domain-average volume fractions. It is readily seen from Fig. 3a that $H_{3 \mathrm{D}}^{\text {one }}$ becomes independent of filter size for $\Delta_{\text {filter }}^{*}>6.7$.

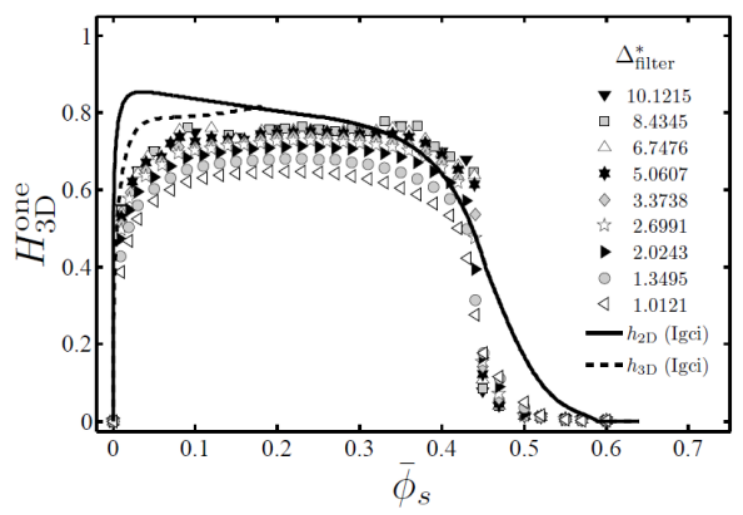

(a)

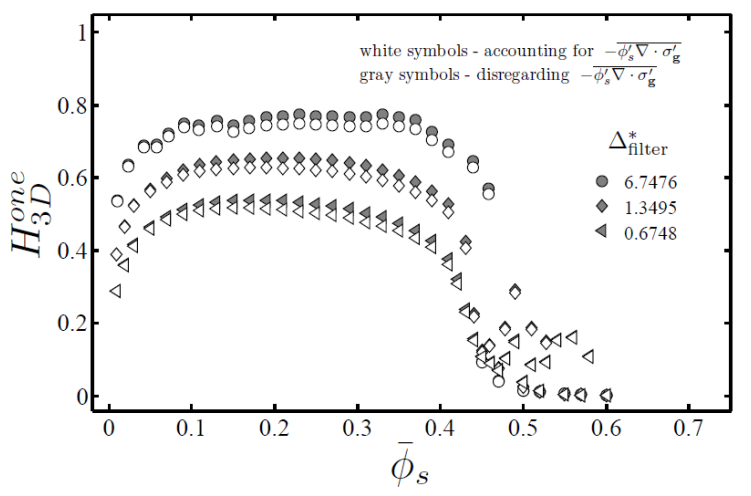

(b)

Fig. 3. (a) Filtered one-marker gas-solid drag correction $H_{3 \mathrm{D}}^{\text {one }}=H_{3 \mathrm{D}}^{\text {one }}\left(\bar{\phi}_{s} ; \Delta_{\text {filter }}^{*}\right)$ versus filtered solids fraction $\bar{\phi}_{s}$ calculated from 3-D simulations for various dimensionless filter sizes $\left(\Delta_{\text {filter }}^{*}=\Delta_{\text {filter }} /\left[v_{t}^{2} / g\right]\right.$ ). The $H_{3 \mathrm{D}}^{\text {one }}$ values are compared with those from previously-developed corrections $h_{2 \mathrm{D}}$ and $h_{3 \mathrm{D}}$, from Igci and Sundaresan (2011a), applicable to very large filter sizes. (b) $H_{3 \mathrm{D}}^{\text {one }}$ with and without the inclusion of $-\overline{\phi_{s}^{\prime} \nabla \cdot \sigma_{g}^{\prime}}$ in $\overline{\boldsymbol{F}}_{g s}$.

\section{Two-marker filtered gas-solid drag}

The one-marker drag correction assumes that the consequences of the inhomogeneous sub-filter scale structure for a specified filter size can be quantified in terms of the filtered volume fraction alone. Recent studies have shown that the addition of filtered slip velocity as a second marker significantly improves the drag correction closure (Milioli et al., 2013). Figure 4 shows the probability distribution functions of dimensionless filtered slip velocity $\tilde{v}_{\text {slip }}^{*}$ at several different filtered particle volume fractions $\bar{\phi}_{s}$. These results represent a composite of data gathered from various simulations. The mean value as well as the 
range and spread of variation of $\tilde{v}_{\text {slip }}^{*}$ systematically vary with $\bar{\phi}_{s}$. For a given $\bar{\phi}_{s}$ value, the variation in $\tilde{v}_{\text {slip }}^{*}$ may be thought of as the consequence of different cluster/bubble sizes and configurations that can exist inside the filtering region, thereby acting as an indicator of heterogeneity. The distributions become narrower at larger $\bar{\phi}_{s}$ values.

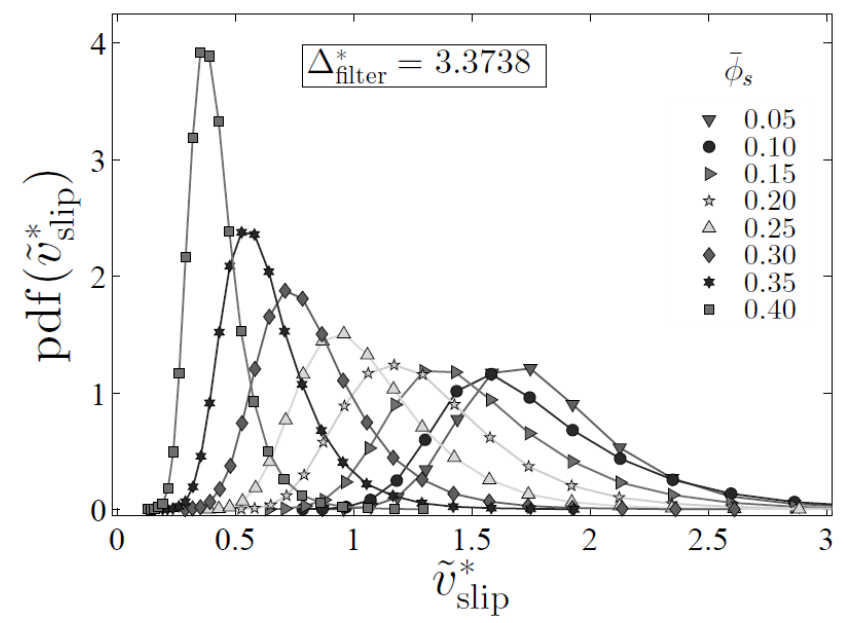

Fig. 4. Probability distribution functions (pdf) of the dimensionless slip velocity $\left(\tilde{v}_{\text {slip }}^{*}\right.$ ) for varying filtered solid fraction $\bar{\phi}_{s}$, shown for a representative filter size $\Delta_{\text {filter }}^{*}=3.3738$.

Figures 5 and 6 present the two-marker filtered drag corrections $H_{3 \mathrm{D}}^{\mathrm{two}}$ calculated from the current 3-D simulation data. From their 2-D results, Milioli et al. (2013) proposed a linear variation of the drag correction with $\bar{\phi}_{s}$ (at each value of $\tilde{v}_{\text {slip }}^{*}$ ). As seen in Fig. 5, $H_{3 \mathrm{D}}^{\mathrm{two}}$ manifests a non-linear dependence on $\bar{\phi}_{s}$, which is captured reasonably well by the new model fits (Eq. 14). The new $H_{3 \mathrm{D}}^{\mathrm{two}}$ values are generally smaller than the 2-D corrections reported by Milioli et al. (2013), consistent with the trend observed for the one-marker model.

The values of $H_{3 \mathrm{D}}^{\mathrm{two}}$ increase with $\tilde{v}_{\text {slip }}^{*}$; i.e., the filtered drag coefficient is smaller at large slip velocities, consistent with 2-D results reported by Milioli et al. (2013). The results in Fig. 5 are re-plotted 
in Fig. 6 as functions of $\tilde{v}_{\text {slip }}^{*}$ for various $\bar{\phi}_{s}$ values. At a specified $\bar{\phi}_{s}$ value, the occurrence of larger $\tilde{v}_{\text {slip }}^{*}$ is attributed to greater sub-filter-scale inhomogeneity and, hence, a reduction in the filtered drag coefficients (i.e., larger $H_{3 \mathrm{D}}^{\mathrm{two}}$ values).

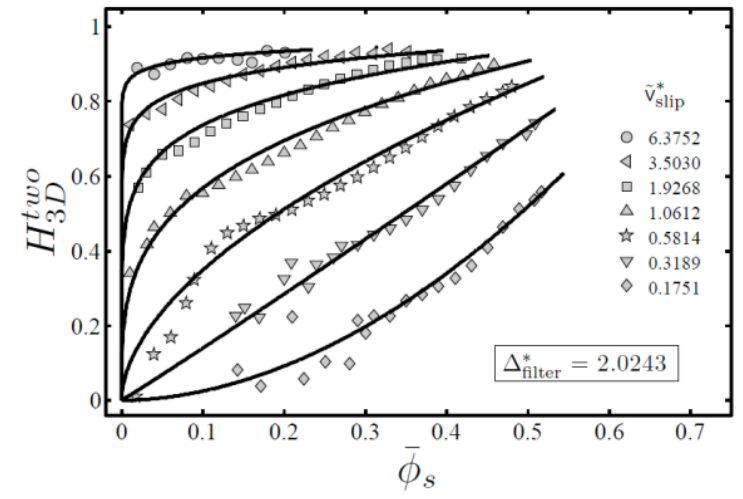

(a)

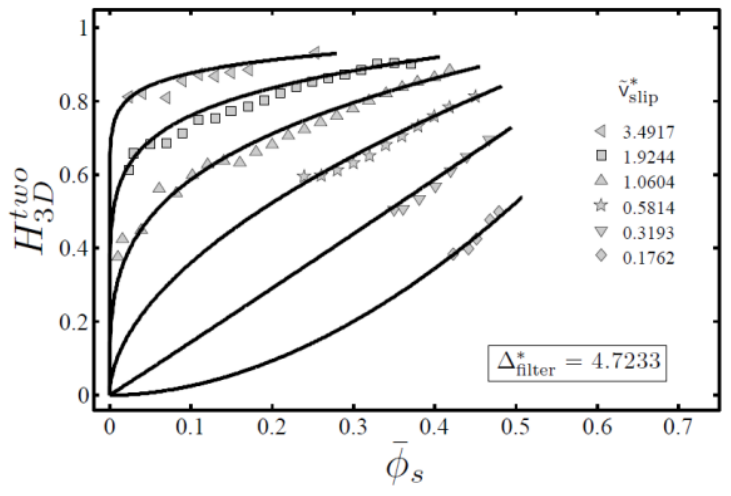

(b)

Fig. 5. Filtered two-marker gas-solid drag correction $H_{3 D}^{t w o}=H_{3 D}^{t w o}\left(\bar{\phi}_{s} ; \widetilde{\mathrm{v}}_{\text {slip }}^{*} ; \Delta_{\text {filter }}^{*}\right)$ versus filtered solids fraction $\bar{\phi}_{s}$ for two representative dimensionless filter sizes: (a) $\Delta_{\text {filter }}^{*}=2.0243$ and (b) $\Delta_{\text {filter }}^{*}=4.7233$. The different symbols represent varying values of the second marker, $\tilde{v}_{\text {slip }}^{*}$. The solid lines are fits for the $H_{3 \mathrm{D}}^{\mathrm{two}}$ model given by Eq. 14 .

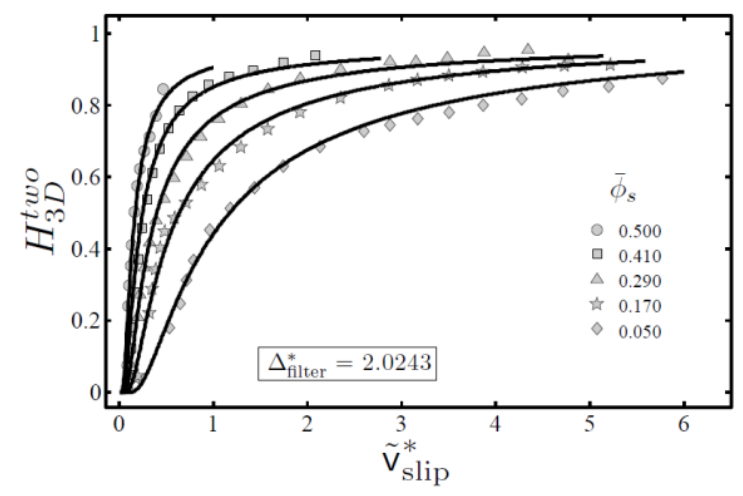

(a)

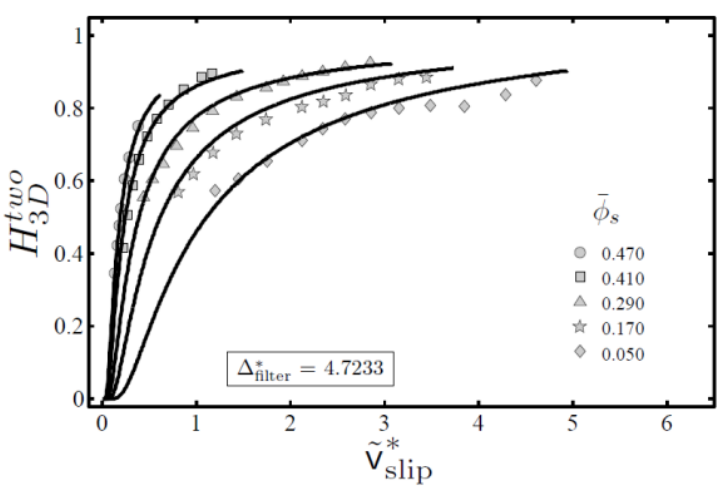

(b)

Fig. 6. Filtered two-marker gas-solid drag correction $H_{3 \mathrm{D}}^{\text {two }}$ versus dimensionless filtered slip velocity ( $\tilde{v}_{\text {slip }}^{*}$ ) for two representative dimensionless filter sizes (a) $\Delta_{\text {filter }}^{*}=2.0243$ and (b) $\Delta_{\text {filter }}^{*}=4.7233$. 
Different symbols represent varying filtered solids fraction $\left(\bar{\phi}_{s}\right)$. The solid lines are the fits given by Eq. 14.

The influence of the filter size $\Delta_{\text {filter }}$ is also captured by the new fits (Eq. 14). The drag correction $H_{3 \mathrm{D}}^{\mathrm{two}}$ increases with filter size, but nearly asymptotes for $\Delta_{\text {filter }}^{*}>6.7$. The filtered drag correction for very large filter sizes, i.e., for device-scale simulations with very-coarse CFD grids, can be obtained by substituting $\Delta_{\text {filter }}^{*} \rightarrow \infty$ in Eq. 14 .

Although the net filtered drag force is defined as $\overline{\boldsymbol{f}}_{g s}-\overline{\phi_{s}^{\prime} \nabla \cdot \boldsymbol{\sigma}_{g}^{\prime}}$, Milioli et al. (2013) did not account for $-\overline{\phi_{s}^{\prime} \nabla \cdot \boldsymbol{\sigma}_{g}^{\prime}}$, as its contribution was deemed to be small compared to that of $\bar{f}_{g s}$. Our 3-D results show that $-\overline{\phi_{s}^{\prime} \nabla \cdot \boldsymbol{\sigma}_{g}^{\prime}}$ contributes very little in the dilute and dense regions; but could be important at intermediate solid fractions (Fig. 7). At its maximum value near $\bar{\phi}_{s} \approx 0.30$, this term constitutes approximately $12 \%$ of the total filtered drag force (also holds true for other filter sizes examined). Our $H_{3 \mathrm{D}}^{\mathrm{two}}$ results include both contributions $\left(\bar{f}_{g s}\right.$ and $-\overline{\phi_{s}^{\prime} \nabla \cdot \boldsymbol{\sigma}_{g}^{\prime}}$ ) to the drag force.

Because the filtered models presented by Milioli et al. (2013), as well as Schneiderbauer and Pirker (2014), do not include the buoyancy fluctuation term $\left(-\overline{\phi_{s}^{\prime} \nabla \cdot \boldsymbol{\sigma}_{g}^{\prime}}\right)$, a direct comparison of the correction factors they present with the current set of models would not be meaningful. Moreover, Schneiderbauer and Pirker (2014) use a 2-D bubbling bed with a frictional constitutive model to derive their sub-grid corrections, as opposed to the full range of solid fractions based on frictionless particles reported by Milioli et al. (2013) and the current work. Therefore, a comparison of the hydrodynamic predictions afforded by the various sub-grid closures would be more appropriate to contrast the different modelsthis will be addressed in a future study. 


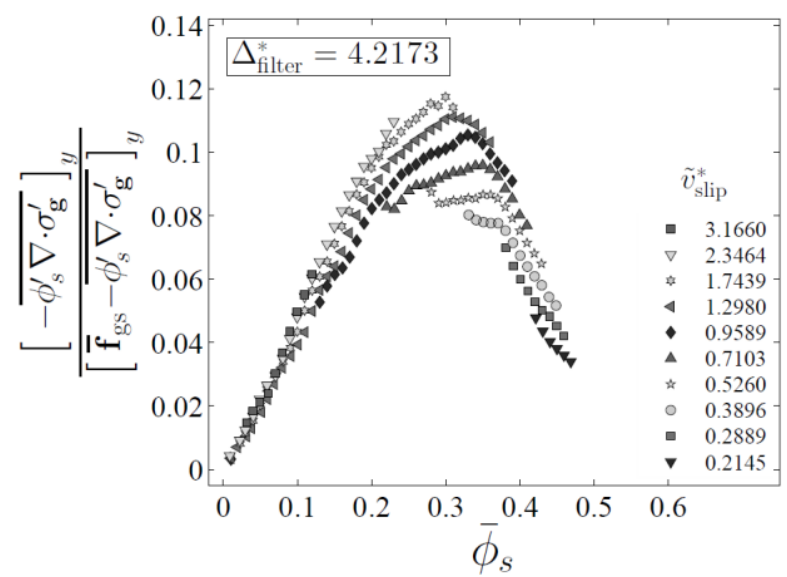

Fig. 7. Fractional contribution of the vertical component of $\left(-\overline{\phi_{s}^{\prime} \nabla \cdot \boldsymbol{\sigma}_{g}^{\prime}}\right)$ to the vertical component of the filtered drag force $\left(\overline{\boldsymbol{f}}_{g s}-\overline{\phi_{s}^{\prime} \nabla \cdot \boldsymbol{\sigma}_{g}^{\prime}}\right)$ as a function of filtered solids fraction $\bar{\phi}_{s}$. Different symbols represent varying dimensionless slip velocity $\tilde{v}_{\text {slip }}^{*}$, and a representative filter size $\Delta_{\text {filter }}^{*}=4.2173$.

\section{Filtered stress models}

Sub-grid stress models for single-phase turbulent flows are often constituted using the scalar filtered rate-of-strain (Pope, 2000). The scalar filtered rates-of-strain, $\overline{S_{i}}=\sqrt{2 \overline{\mathbf{s}}_{i}: \overline{\mathbf{s}}_{i}}, i=s, g$, have been used to constitute the filtered pressure and viscosities in multiphase flows as well (Milioli et al., 2013). From their 2-D simulations, Milioli et al. (2013) found a clear dependence of the dimensionless filtered rates-ofstrain $\bar{S}_{i}^{*}=\bar{S}_{i} /\left[g / v_{t}\right]$ on $\Delta_{\text {filter }}^{*}$ and $\bar{\phi}_{s}$, but not on $\tilde{v}_{\text {slip }}^{*}$. In contrast, the present 3-D simulation data reveal an unmistakable dependence of $\bar{S}_{s}^{*}$ and $\bar{S}_{g}^{*}$ on $\tilde{v}_{\text {slip }}^{*}$ (Fig. 8). Furthermore, Figs. 8a and 8b are very similar, both qualitatively and quantitatively. Closures for $\bar{S}_{s}^{*}$ or $\bar{S}_{g}^{*}$ are not needed to solve the filtered conservation equations; ergo, fits for $\bar{S}_{s}^{*}$ and $\bar{S}_{g}^{*}$ are not developed. Figure 8 suggests that $\bar{S}_{s}^{*}$ or $\bar{S}_{g}^{*}$ could serve as a second marker (instead of $\tilde{v}_{\text {slip }}^{*}$ ); thus, $\bar{S}_{s}^{*}\left(\right.$ or $\bar{S}_{g}^{*}$ ) based closures, which also include $\bar{\phi}_{s}$ and $\Delta_{\text {filter }}^{*}$, can be thought of as two-marker models as well. Note that slip velocity could have been used as the second marker to construct the stress models, since the filtered rate-of-strain $\left(\bar{S}_{s}^{*}\right.$ and $\left.\bar{S}_{g}^{*}\right)$ and $\tilde{v}_{\text {slip }}^{*}$ 
are related. Eventually, we decided in favor of simpler closures using $\bar{S}_{s}^{*}$ and $\bar{S}_{g}^{*}$, similar to the sub-grid expressions encountered in single-phase turbulence. We have not pursued this line of inquiry in this study, i.e., probing alternate choices for the second marker, which could also include the (anisotropic) meso-scale velocity gradients.

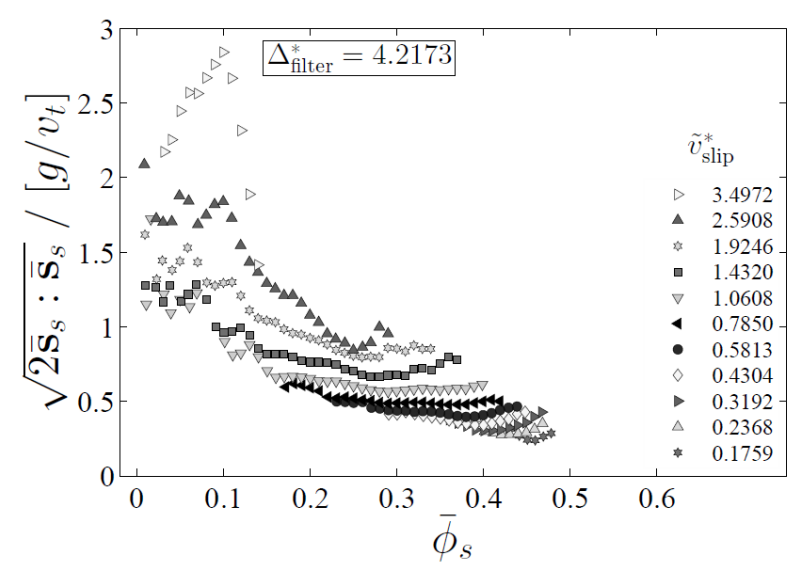

(a)

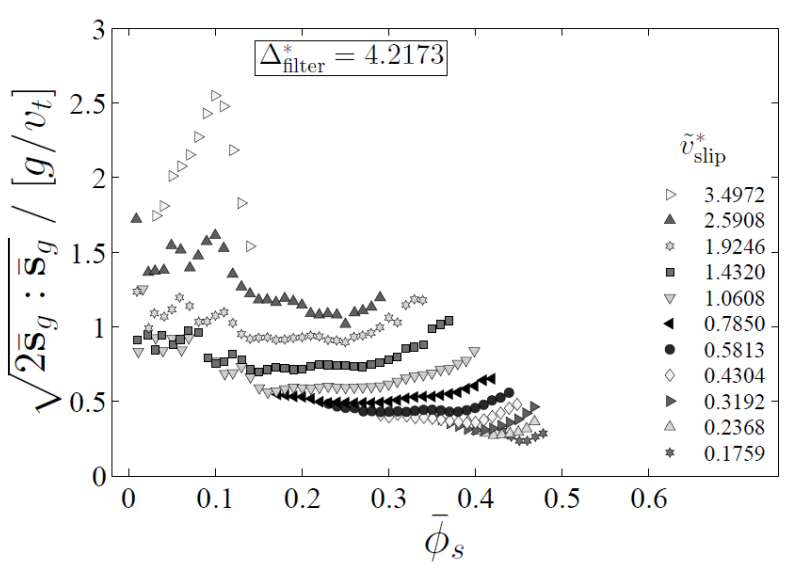

(b)

Fig. 8. Filtered scalar rate-of-strain $\bar{S}_{i}=\sqrt{2 \overline{\mathbf{s}}_{i}: \overline{\mathbf{s}}_{i}}(i=s, g)$, made dimensionless by $g / v_{t}$, for (a) particle phase and (b) gas phase as a function of filtered solid fraction $\bar{\phi}_{s}$. Different symbols represent varying slip velocity $\tilde{v}_{\text {slip }}^{*}$. Measurements are shown for a representative filter size $\Delta_{\text {filter }}^{*}=4.2173$.

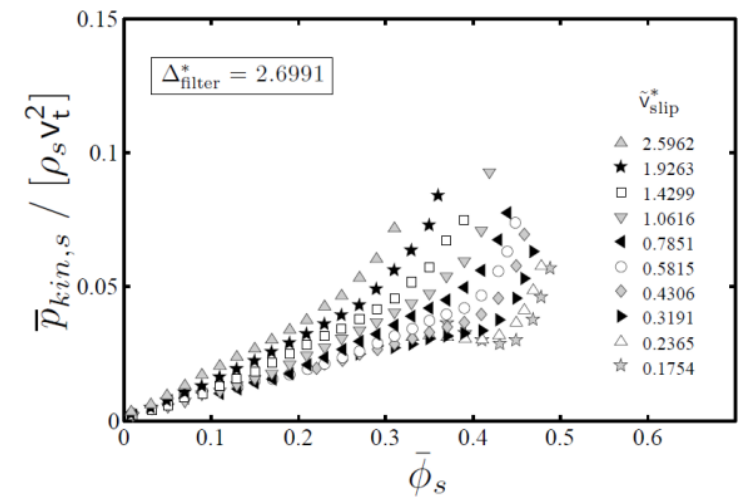

(a)

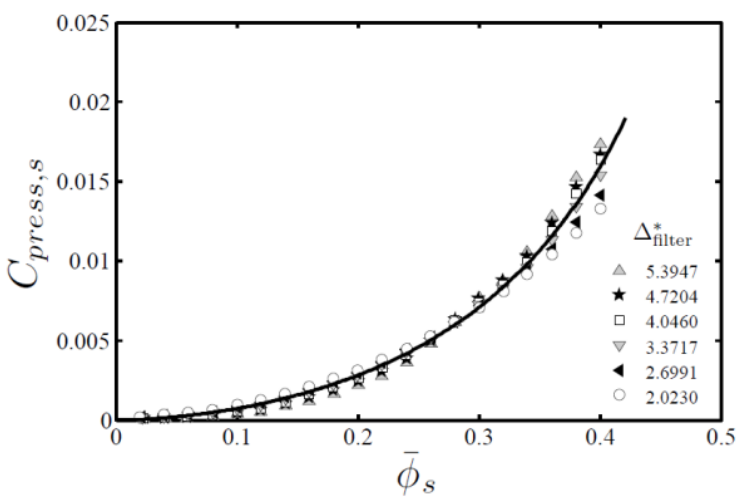

(b)

Fig. 9. (a) Filtered kinetic-theory pressure of the solid phase, $\bar{p}_{k i n, s}$, made dimensionless using characteristic stress $\rho_{s} v_{t}^{2}$, as a function of filtered solids fraction $\bar{\phi}_{s}$. Results are shown for a 
representative filter size $\Delta_{\text {filter }}^{*}=2.6991$. Different symbols represent varying $\tilde{v}_{\text {slip }}^{*}$ values. (b) Variation of $C_{\text {press,s }}$ with filtered solids fraction $\bar{\phi}_{s}$ for different filter sizes. The solid line represents Eq. 15.

In the filtered stress analysis described below, we consider filtered micro-scale and meso-scale pressures and viscosities, as well as meso-scale normal-stress differences. All these filtered quantities were found to depend on both markers $\left(\bar{\phi}_{s}\right.$ and $\left.\tilde{v}_{\text {slip }}^{*}\right)$. However, in view of our earlier observation that coarse dynamics of fluidized suspensions are largely influenced by the filtered drag closures, with filtered stress playing only a secondary role, we deem one-marker closures for the stresses to be adequate.

The solid phase pressure is comprised of two components (refer to Eq. 10): the filtered microscopic (kinetic-theory) solid pressure, $\bar{p}_{k i n, s}=\left[\bar{p}_{s}-\overline{\eta \mu_{b}\left(\nabla \cdot v_{s}\right)}\right]$, and the filtered meso-scale pressure, defined as $\bar{p}_{\text {meso,s }}=\frac{1}{3} \operatorname{tr}\left[\rho_{s} \overline{\phi_{s} \boldsymbol{v}_{s}^{\prime} \boldsymbol{v}_{s}^{\prime}}\right]$. At the packing limit, the filtered microscopic pressure diverges whereas the filtered mesoscopic pressure approaches zero and, therefore, separate closures for the two components would be more desirable.

The dimensionless filtered kinetic-theory pressure of the solid phase $\bar{p}_{k i n, s} / \rho_{s} v_{t}^{2}$ is a function of $\bar{\phi}_{s}$ and $\tilde{v}_{\text {slip }}^{*}$, and generally increases with respect to both variables (Fig. 9a). A simple scaling model captures the dependence of $\bar{p}_{k i n, s}$ on $\bar{\phi}_{s}$ and $\tilde{v}_{\text {slip }}^{*}$, given as: $C_{\text {press }, s}=\bar{p}_{k i n, s} /\left[\rho_{s}\left(\Delta_{\text {filter }}\right)^{2} \bar{S}_{s}^{2}\right]$ (see Eq. 15), as demonstrated in Fig. $9 b$.

The dimensionless filtered kinetic-theory viscosity of the solid phase, defined as $\bar{\mu}_{s}=\left|\bar{\sigma}_{s}\right|_{\mathrm{i} \neq \mathrm{j}} / \bar{S}_{s, i \neq j}$, is also a function of $\bar{\phi}_{s}$ and $\tilde{v}_{\text {slip }}^{*}$, and generally increases with respect to both variables (Fig. 10a). (As discussed later, in view of appreciable meso-scale normal stress anisotropy, filtered viscosity is estimated by focusing on off-diagonal terms. To be consistent, we have used the same analysis procedure for the filtered micro-scale viscosity, and hence the $i j$ restriction.) Figure $10 \mathrm{~b}$ shows the dependence of 
$C_{v i s c, s}=\bar{\mu}_{s} /\left[\rho_{s}\left(\frac{g}{v_{t}^{2}}\right)^{-6 / 7}\left(\Delta_{\text {filter }}\right)^{8 / 7} \bar{S}_{s}\right]$ on $\bar{\phi}_{s}$; Eq. (16) then provides a convenient way of quantifying the filtered micro-scale viscosity results.

The filtered meso-scale pressure of the solid phase $\bar{p}_{\text {meso }, s}=\frac{1}{3} \operatorname{tr}\left[\rho_{s} \overline{\phi_{s} \boldsymbol{v}_{s}^{\prime} \boldsymbol{v}^{\prime}}\right]$ represents a Reynolds-stress-like contribution, which arises from sub-filter-scale velocity fluctuations (refer to Eq. 10). Typically, $\bar{p}_{m e s o, s}$ values are much larger than $\bar{p}_{k i n, s}$, especially for large filter sizes (Agrawal et al., 2001). As such, many previous studies have constituted the net solids pressure $\left(\bar{p}_{k i n, s}+\bar{p}_{m e s o, s}\right)$ using a single expression (Igci et al., 2008; Igci and Sundaresan, 2011a) or chosen to model only the meso-scale pressure $\bar{p}_{m e s o, s}$ (Milioli et al., 2013). The dimensionless meso-scale pressure $\bar{p}_{m e s o, s} / \rho_{s} v_{t}^{2}$ is a function of $\bar{\phi}_{s}$ as well as $\tilde{v}_{\text {slip }}^{*}$ (Fig. 11a). This finding is in contrast with the 2-D results of Milioli et al. (2013), who did not find any clear dependence on $\tilde{v}_{\text {slip }}^{*}$. Clearly, 2-D simulations fail to capture some of the characteristics of the 3-D flow structures. Figure $11 \mathrm{~b}$ shows the dependence of $C_{\text {press }, \text { meso }, s}=\bar{p}_{\text {meso }, s} /\left[\rho_{s}\left(\frac{g}{v_{t}^{2}}\right)^{3 / 7}\left(\Delta_{\text {filter }}\right)^{17 / 7} \bar{S}_{s}^{2}\right]$ on $\bar{\phi}_{s}$, which shows that $C_{\text {press }, \text { meso }, s}$ can be approximated as a univariate function of $\bar{\phi}_{s}$, Thus, the fit given by Eq. (17) can be used as a closure for the filtered meso-scale pressure. 


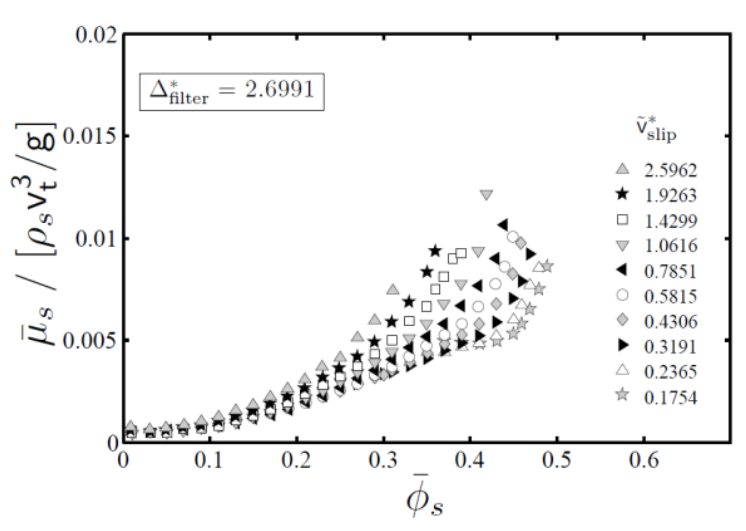

(a)

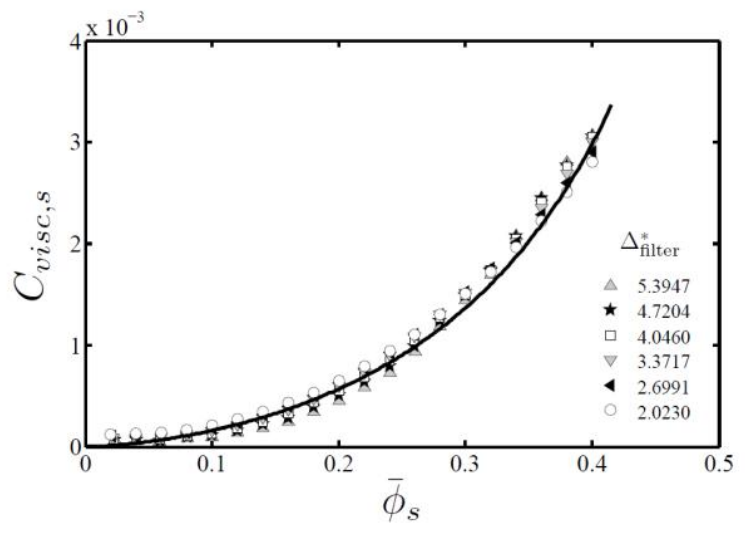

(b)

Fig. 10. (a) Filtered kinetic-theory viscosity of the solid phase, $\bar{\mu}_{s}=\left|\overline{\boldsymbol{\sigma}}_{s}\right|_{\mathrm{i} \neq \mathrm{j}} / \bar{S}_{s, i \neq j}$, made dimensionless using the characteristic amount $\rho_{s} v_{t}^{3} / g$, as a function of filtered solids fraction $\bar{\phi}_{s}$. Results are shown for a representative filter size $\Delta_{\text {filter }}^{*}=2.6991$. Different symbols represent varying $\tilde{v}_{\text {slip }}^{*}$ values. (b) Variation of $C_{v i s c, s}$ with filtered solids fraction $\bar{\phi}_{s}$ for different filter sizes. The solid line represents Eq. 16.

The dependence of filtered meso-scale viscosity of the solid phase, $\bar{\mu}_{m e s o, s}=\left|\rho_{s} \overline{\phi_{s} v_{s}^{\prime} v_{s}^{\prime}}\right|_{i \neq \mathrm{j}} / \bar{S}_{s, i \neq j}$ with $\bar{\phi}_{s}$ is shown in Fig. 12a for several $\tilde{v}_{\text {slip }}^{*}$ and a representative filter size. The variation of $C_{\text {visc,meso }, s}=\bar{\mu}_{\text {meso }, s} /\left[\rho_{s}\left(\frac{g}{v_{t}^{2}}\right)^{-2 / 7}\left(\Delta_{\text {filter }}\right)^{12 / 7} \bar{S}_{s}\right]$ with $\bar{\phi}_{s}$ is presented it Fig. $12 \mathrm{~b}$ and, as before, the fit given by Eq. (18) then provides a model for the meso-scale viscosity. 


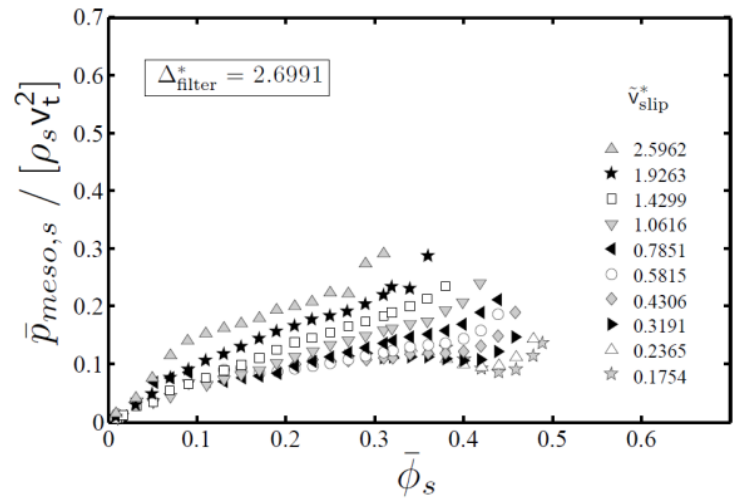

(a)

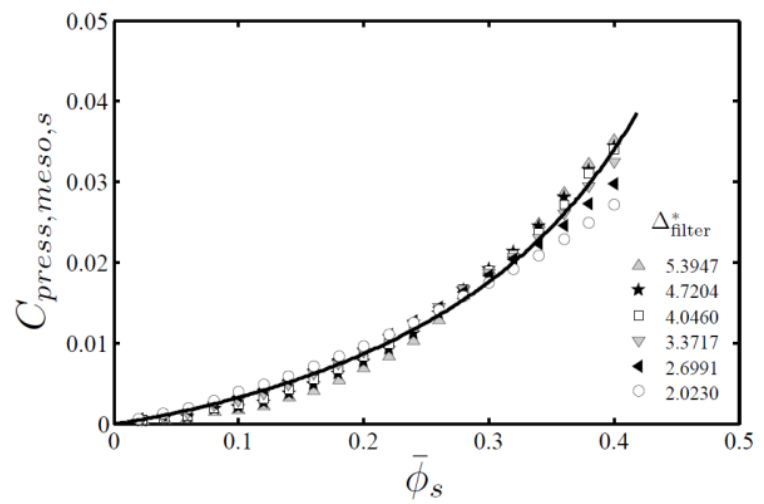

(b)

Fig. 11. (a) Filtered meso-scale pressure of the solid phase, $\bar{p}_{\text {meso }, s}=\frac{1}{3} \operatorname{tr}\left[\rho_{s} \overline{\boldsymbol{\phi}_{s} \boldsymbol{v}_{s}^{\prime} \boldsymbol{v}^{\prime}}\right]$, made dimensionless using $\rho_{s} v_{t}^{2}$, as a function of filtered solids fraction $\bar{\phi}_{s}$. Results are shown for a representative filter size $\Delta_{\text {filter }}^{*}=2.6991$. Different symbols represent varying $\tilde{v}_{\text {slip }}^{*}$ values. (b) Variation of $C_{\text {press,meso,s }}$ with filtered solids fraction $\bar{\phi}_{s}$ for different filter sizes. The solid line represents Eq. 17.

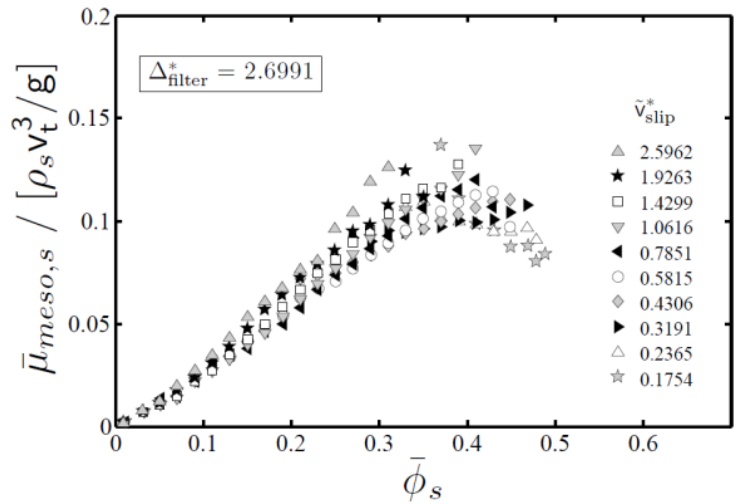

(a)

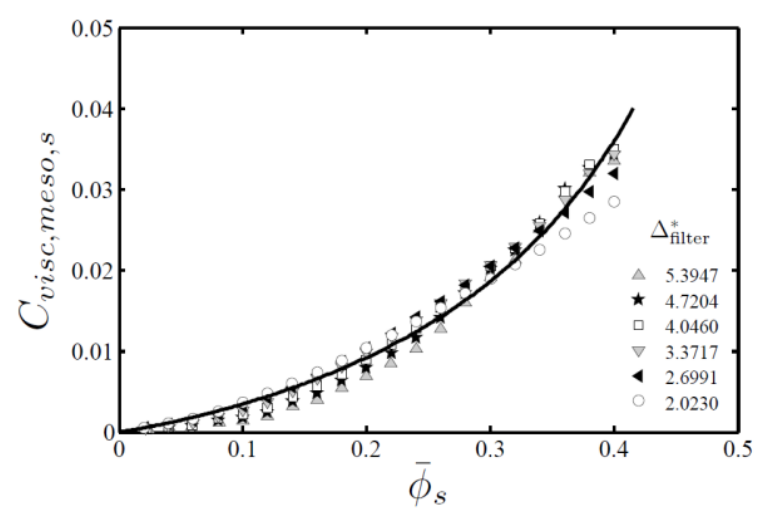

(b)

Fig. 12. (a) Filtered meso-scale viscosity of the solid phase, $\bar{\mu}_{m e s o, s}=\left|\rho_{s} \overline{\phi_{s} v_{s}^{\prime} v_{s}^{\prime}}\right|_{\mathrm{i} \neq \mathrm{j}} / \bar{S}_{s, i \neq j}$, made dimensionless using $\rho_{s} v_{t}^{3} / g$, as a function of filtered solids fraction $\bar{\phi}_{s}$. Results are shown for a representative filter size $\Delta_{\text {filter }}^{*}=2.6991$. Different symbols represent varying $\tilde{v}_{\text {slip }}^{*}$ values. (b) Variation of $C_{\text {visc,meso,s }}$ with filtered solids fraction $\bar{\phi}_{s}$ for different filter sizes. The solid line represents Eq. 18. 
Anisotropy is well known in granular flows (Campbell, 1997; Natarajan et al., 1995) as well as in gas-particle flows (Agrawal et al., 2001; Holland et al., 2008; Holloway et al., 2012). In the present simulations, anisotropy in the solids-phase normal stresses is observed owing to the vertical gravitational force and preferential axial direction of gas/solid flow. The anisotropy, characterized by the (dimensionless) normal stress differences, exists between the vertical and transverse components, i.e., the values of $\quad \bar{N}_{s, 1}^{*}=\left(\bar{\Sigma}_{s, y y}-\bar{\Sigma}_{s, x x}\right) /\left(\rho_{s} v_{t}^{2}\right)\left[\approx\left(\bar{\Sigma}_{s, y y}-\bar{\Sigma}_{s, z z}\right) /\left(\rho_{s} v_{t}^{2}\right)\right] \quad$ are non-zero, while $\bar{N}_{s, 2}^{*}=\left(\bar{\Sigma}_{s, x x}-\bar{\Sigma}_{s, z z}\right) /\left(\rho_{s} v_{t}^{2}\right)$ is practically zero. Figure 13a shows these trends for a particular filter size ( $\left.\Delta_{\text {filter }}^{*}=2.6991\right)$

Although the normal stress difference calculations consider the total contributions from all stress components (refer to Eq. 10), the meso-scale solids pressure is found to be the primary source of anisotropy (also reported by Agrawal et al., 2001). Therefore, a constitutive model for the normal-stress anisotropy is developed based on $\bar{p}_{m e s o, s}$. Figure $13 \mathrm{~b}$ shows $\bar{N}_{s, 1}^{*}$ scaled with $\bar{p}_{m e s o, s}$ for a particular filter size. While a second-marker dependence is observed, we are not aware of any flow phenomenon in fluidized beds that have been attributed to normal stress differences, and so the usefulness of including such differences in a filtered model remains to be demonstrated. With this in mind, we sought a simple one-marker model. Figure $13 \mathrm{c}$ shows that the scaled normal stress difference is essentially independent of filter size and can be captured by a simple relation (Eq. 20).

The filtered gas-phase meso-scale pressure, $\quad \bar{p}_{m e s o, g}=\frac{1}{3} \operatorname{tr}\left[\rho_{g} \overline{\phi_{g} \boldsymbol{v}_{g}^{\prime} \boldsymbol{v}_{g}^{\prime}}\right]$, and viscosity, $\bar{\mu}_{m e s o, g}=\left|\rho_{g} \overline{\phi_{g} v_{g}^{\prime} \boldsymbol{v}_{g}^{\prime}}\right|_{\mathrm{i} \neq \mathrm{j}} / \bar{S}_{g, i \neq j}$, shown in Figs. 14 and 15, respectively, are at least an order of magnitude smaller than their particle phase counterparts. Nevertheless, correlations are also provided for these quantities for the sake of completeness, using closures similar in form as presented previously. The variation of $C_{\text {press,meso }, g}=\bar{p}_{\text {meso }, g} /\left[\rho_{g}\left(\frac{g}{v_{t}^{2}}\right)^{5 / 7}\left(\Delta_{\text {filter }}\right)^{19 / 7} \bar{S}_{g}^{2}\right]$ with filtered solids fraction $\bar{\phi}_{s}$ for 
different filter sizes, shown in Fig. 14, is adequately fit by Eq. 22. Similarly, a model for $C_{\text {visc,meso,g }}=\bar{\mu}_{\text {meso, },} /\left[\rho_{g}\left(\Delta_{\text {filter }}\right)^{2} \bar{S}_{g}\right]$ as a function of $\bar{\phi}_{s}$ is captured by Eq. 23 ; represented by the solid line in Fig. 15.

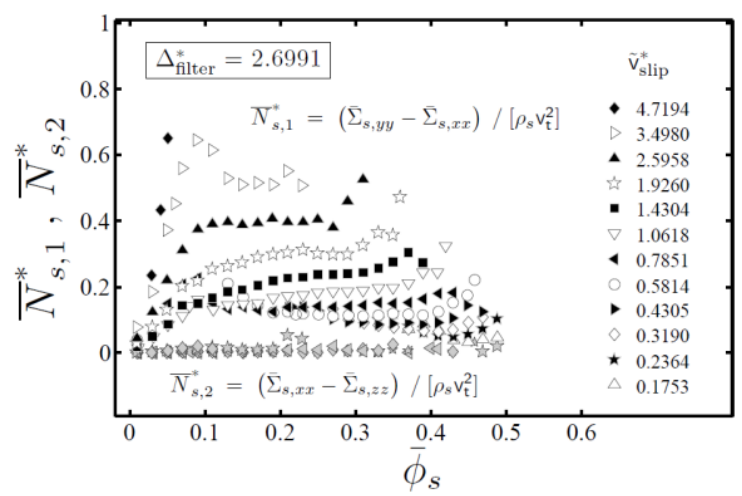

(a)

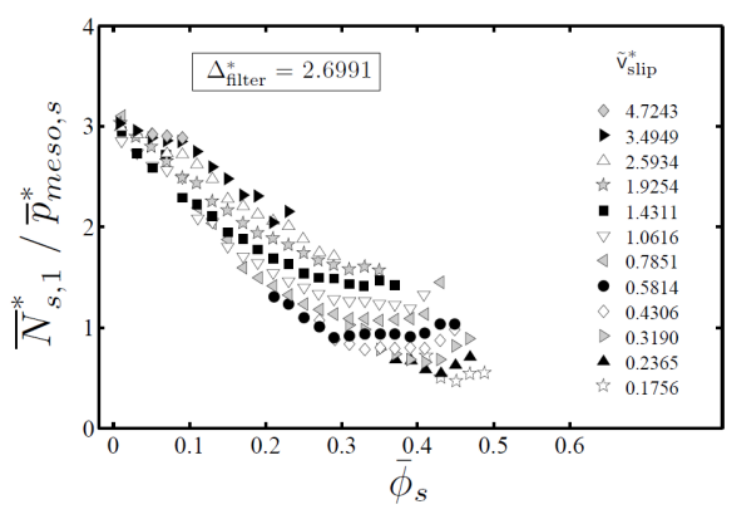

(b)

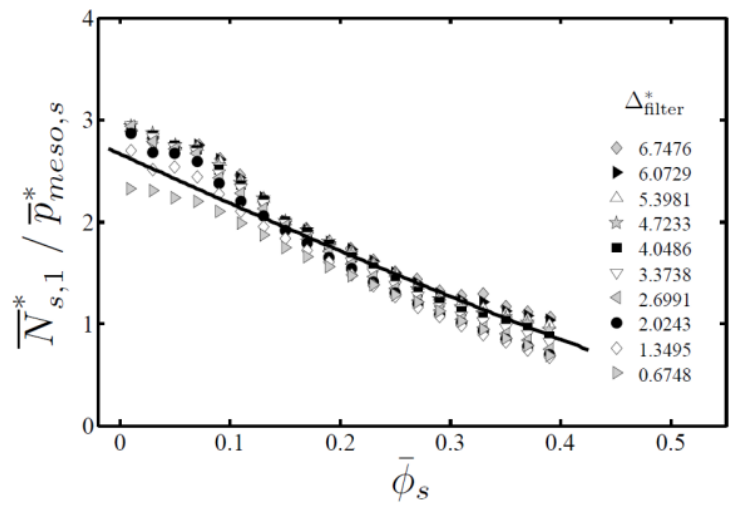

(c)

Fig. 13. (a) Filtered stress anisotropy expressed via the normal stress differences $\left(\bar{N}_{s, 1}^{*}\right.$ and $\left.\bar{N}_{s, 2}^{*}\right)$, made dimensionless by $\rho_{s} v_{t}^{2}$, as a function of solid fraction $\bar{\phi}_{s}$. The $\bar{N}_{s, 1}^{*}$ values are shown using white- and black-colored markers, and the $\bar{N}_{s, 2}^{*}$ values (which are practically zero) are shown using markers colored gray. (b) $\bar{N}_{s, 1}^{*}$ values scaled with $\bar{p}_{m e s o, s}$. Results are shown for a particular filter size $\Delta_{\text {filter }}^{*}=2.6991$. Different symbols in (a) and (b) represent varying $\tilde{v}_{\text {slip }}^{*}$ values. (c) One-marker approach for the scaled $\bar{N}_{s, 1}^{*}$ values for various filter sizes; the solid line represents Eq. 20. 


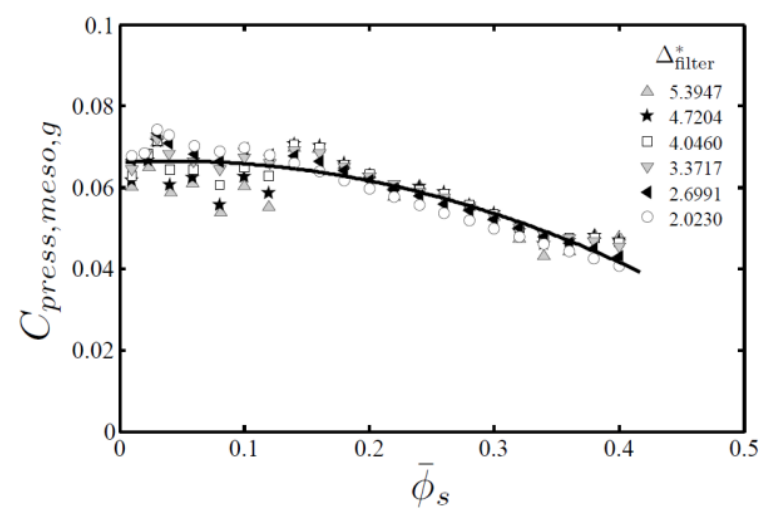

Fig. 14. Variation of $C_{\text {press,meso,g }}$ with $\bar{\phi}_{s}$. Different symbols refer to different dimensionless filter sizes. The solid line represents Eq. 22.

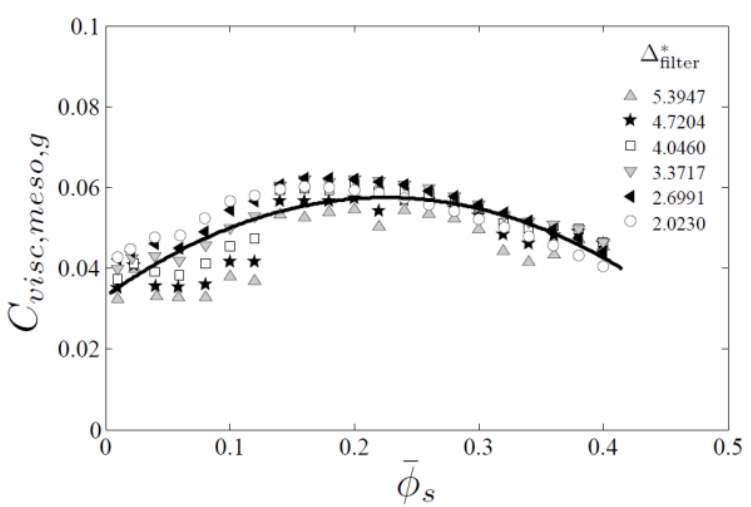

Fig. 15. Variation of $C_{\text {visc,meso,g }}$ with $\bar{\phi}_{s}$. Different symbols refer to different dimensionless filter sizes. The solid line represents Eq. 23

\section{Scaling with particle properties}

The filtered corrections developed thus far are based on the material properties of particles that are being developed for carbon-capture applications (Miller et al., 2014; Sarkar et al., 2014a). These carboncapture (CC) particles are larger and lighter $\left(\mathrm{CC}: 150 \mu \mathrm{m}, 441 \mathrm{~kg} / \mathrm{m}^{3}\right)$ compared to the fluid-catalyticcracking particles (FCC: $75 \mu \mathrm{m}, 1500 \mathrm{~kg} / \mathrm{m}^{3}$ ) studied by previous authors (Igci et al., 2008; Igci and Sundaresan, 2011a; Milioli et al., 2013; Parmentier et al., 2012). Igci (2011) found that their dimensionless scaling relationships, which are also used in the present work (refer to Table 1), capture the 
influence of particle size and density on the filtered corrections. However, their investigation was limited to a small range $\left(50 \mu \mathrm{m} \leq d_{p} \leq 100 \mu \mathrm{m}\right)$ around the nominal values for FCC particles $\left(d_{p}=75 \mu \mathrm{m}\right)$.

To further investigate the influence of particle properties, a limited number of periodic-cell simulations with FCC particles were performed in the present study for $\bar{\phi}_{s}=0.10,0.20$, and 0.30 . Only the particle size and density are adjusted in these new simulations, the values listed in Table 1 are retained for all other parameters. The CFD grid size is also refined such that the resolution is maintained at $\Delta_{\text {grid }}=8.33 d_{p}$. The filtered corrections derived from these FCC-particle simulations are compared against the relationships presented above.

The drag corrections deduced from simulations with the two different particles match well at lower slip velocities but deviate at higher $\tilde{v}_{\text {slip }}^{*}$ values (Fig. 16). This suggests that the filtered closures can be improved by introducing an additional dependence on particle Froude number $F r_{p}=v_{t}^{2} /\left(g d_{p}\right)$.

Both CC and FCC particles lead to comparable filtered kinetic-theory pressure and viscosity coefficients of the solid phase $C_{\text {press,s }}$ and $C_{v i s c, s}$ (Fig. 17), and filtered meso-scale pressure and viscosity coefficients of the solid phase, $C_{\text {press,meso,s }}$ and $C_{\text {visc,meso,s }}$ (Fig. 18). The filtered meso-scale pressure and viscosity coefficients of the gas phase, $C_{\text {press,meso,g }}$ and $C_{\text {visc,meso,g }}$ (Fig. 19) reveal some departure. As noted earlier, coarse dynamics of fluidized beds are most influenced by the filtered drag correction; the filtered particle-phase stresses play a secondary role, and the filtered gas-phase stresses have an even lesser impact. Hence, the level of agreement seen in Figs. 17-19 is deemed adequate and future efforts should focus on refinement of the drag correction to capture the particle-Froude-number dependence. Even though some differences in filtered-drag coefficient between CC and FCC particles are observed in Fig. 16, we will show in Sec. 8 that our filtered-model predictions (which are constructed from CCparticle simulations) agree very well with experimental data provided by NETL/PSRI (which used FCC particles). Therefore, the sub-grid drag model presented in this work for the CC particles may be applied, 
at least as a first approximation, for FCC particles as well, and to particles with $F r_{p}$ values intermediate to these two types.

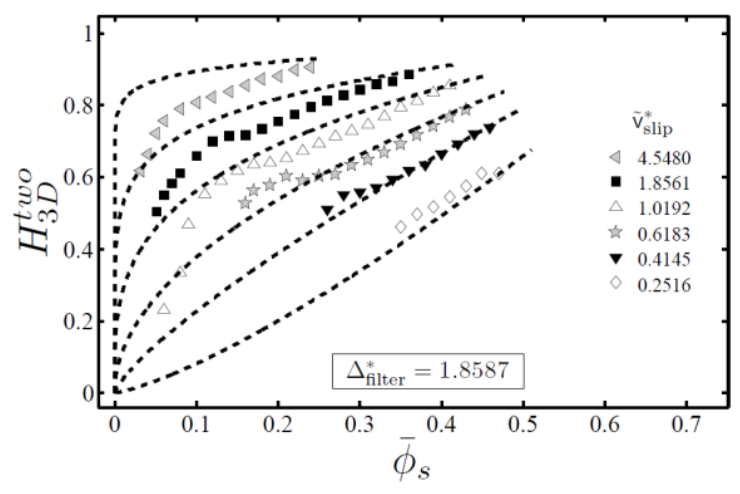

(a)

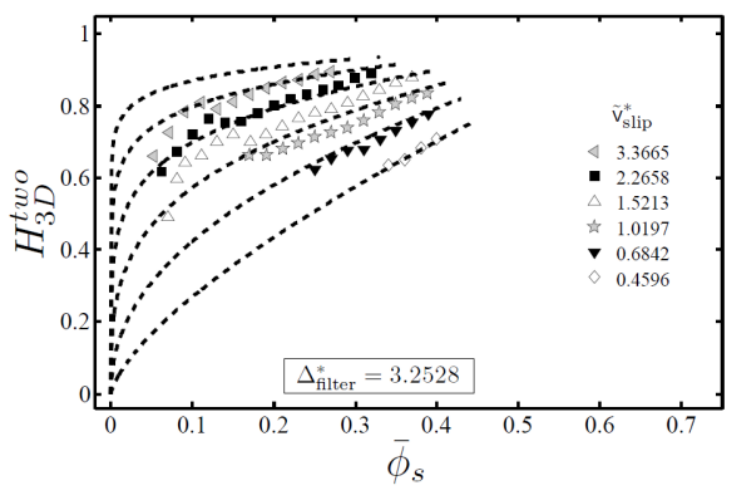

(b)

Fig. 16. Filtered drag correction $H_{3 \mathrm{D}}^{\text {two }}$ derived using FCC particles $\left(75 \mu \mathrm{m}, 1500 \mathrm{~kg} / \mathrm{m}^{3}\right)$, shown using data markers. The corresponding dashed lines represent the model given by Eq. 14, which was derived for CC particles $\left(150 \mu \mathrm{m}, 441 \mathrm{~kg} / \mathrm{m}^{3}\right)$. Comparisons are shown for two representative filter sizes (a) $\Delta_{\text {filter }}^{*}=1.8587$ and (b) $\Delta_{\text {filter }}^{*}=3.2528$. Varying values of $\tilde{v}_{\text {slip }}^{*}$ are showing using different marker types.

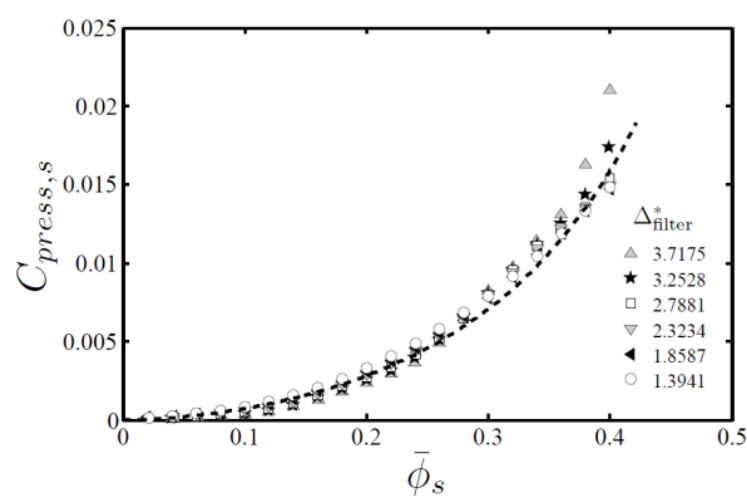

(a)

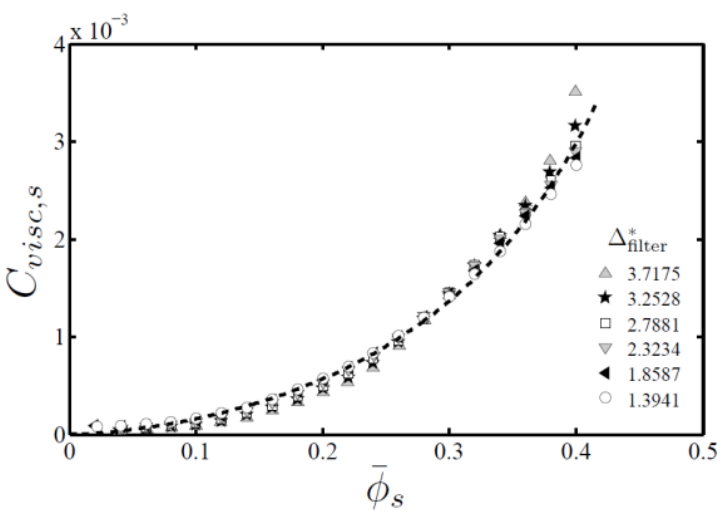

(b)

Fig. 17. Filtered kinetic-theory coefficients for (a) pressure $C_{\text {press,s }}$ and (b) viscosity $C_{v i s c, s}$ of the solid phase, derived using FCC particles $\left(75 \mu \mathrm{m}, 1500 \mathrm{~kg} / \mathrm{m}^{3}\right)$ shown by the data markers. The dashed lines show the model fits for $C_{\text {press,s }}$ and $C_{\text {visc,s }}$ given by Eqs. 15 and 16, respectively, which were derived using CC particles $\left(150 \mu \mathrm{m}, 441 \mathrm{~kg} / \mathrm{m}^{3}\right)$. Different markers represent varying filter sizes. 


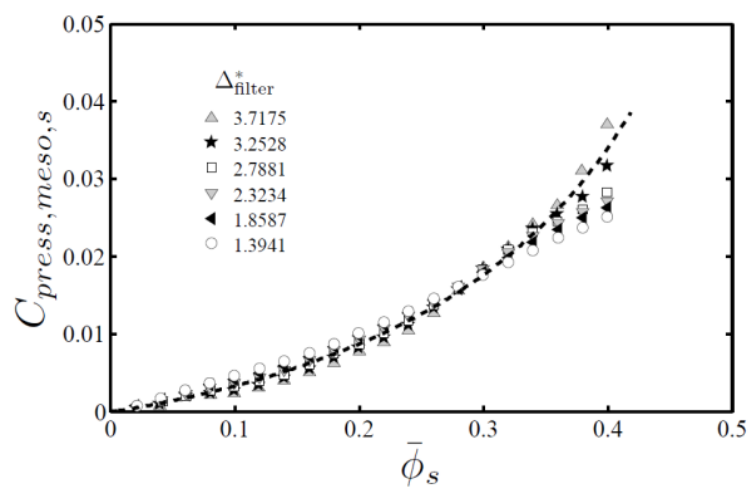

(a)

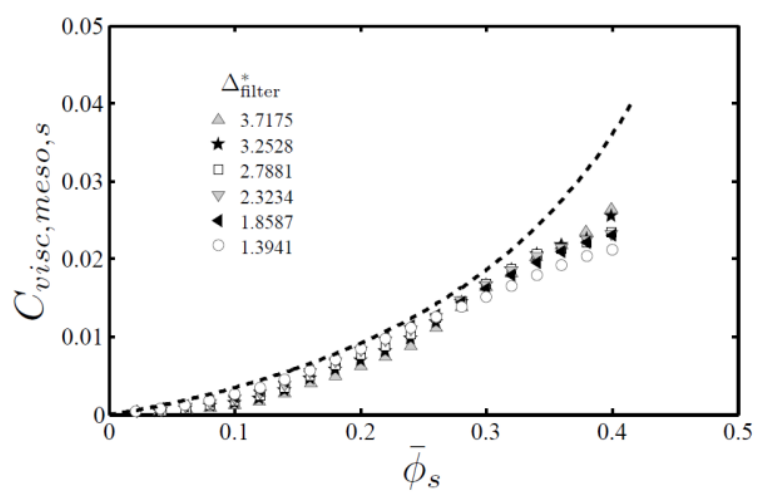

(b)

Fig. 18. Filtered meso-scale coefficients for (a) pressure $C_{\text {press,meso,s }}$ and (b) viscosity $C_{\text {visc,meso,s }}$ of the solid phase, derived using FCC particles $\left(75 \mu \mathrm{m}, 1500 \mathrm{~kg} / \mathrm{m}^{3}\right)$ shown by the data markers. The dashed lines show the model fits for $C_{\text {press,meso,s }}$ and $C_{\text {visc,meso,s }}$ given by Eqs. 17 and 18, respectively, which were derived using CC particles $\left(150 \mu \mathrm{m}, 441 \mathrm{~kg} / \mathrm{m}^{3}\right)$. Different markers represent varying filter sizes.

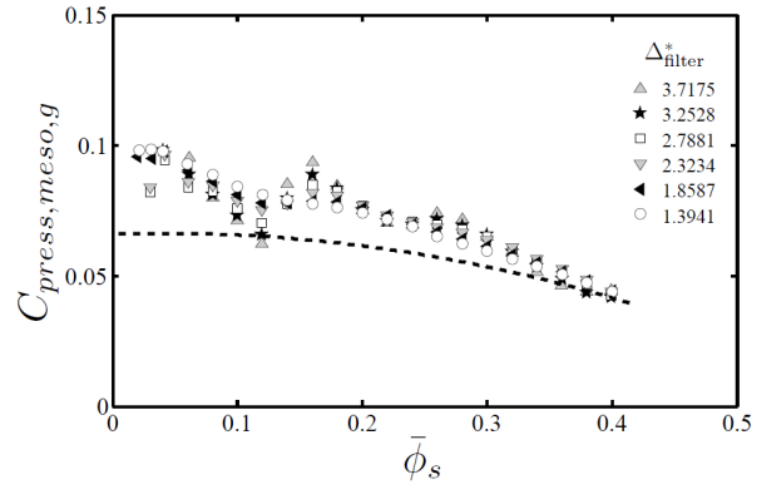

(a)

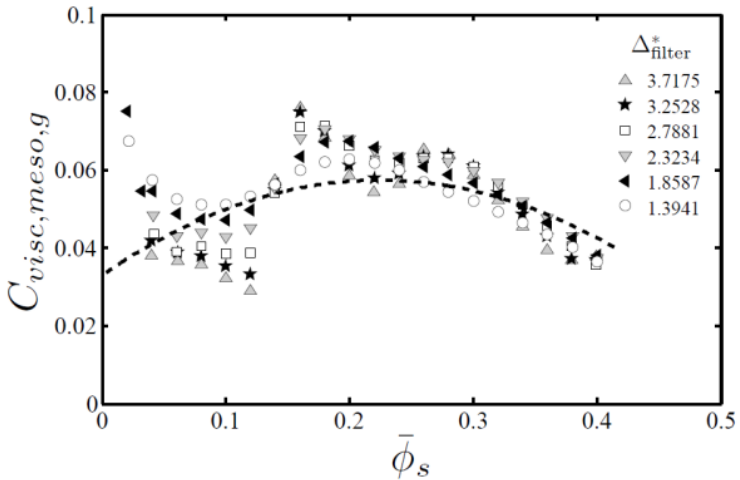

(b)

Fig. 19. Filtered coefficients for the meso-scale (a) pressure $C_{\text {press,meso,g }}$ and (b) viscosity $C_{\text {visc,meso }, g}$ of the gas phase, derived using FCC particles $\left(75 \mu \mathrm{m}, 1500 \mathrm{~kg} / \mathrm{m}^{3}\right)$ shown by the data markers. The dashed lines show the model fits for $C_{\text {press,meso,g }}$ and $C_{\text {visc,meso,g }}$ given by Eqs. 22 and 23, respectively, which were derived using CC particles $\left(150 \mu \mathrm{m}, 441 \mathrm{~kg} / \mathrm{m}^{3}\right)$. Different markers represent varying filter sizes.

\section{Validation test}

As a test problem to examine the new filtered closures presented in this work, we consider the NETL/PSRI bubbling fluidized bed challenge problem (Shadle et al., 2011). Information on the 
challenge problem including system geometry, dimensions, operating conditions, and instrumentation can be accessed on the NETL website (https://mfix.netl.doe.gov). This test problem has been a subject of a previous simulation study from our group (Ozarkar et al., 2015) where the effect of grid topology and different constitutive models were explored; readers are referred to this work for further information.

The test unit used to gather experimental data consists of a fluidized bed section (with 0.91-m diameter and 7.09-m height), primary and secondary cyclones, and two different types of gas distributors: ring or pipe manifold sparger; see Ozarkar et al. (2015) for a schematic diagram. In the NETL/PSRI experiments, FCC particles (with density of $1489 \mathrm{~kg} / \mathrm{m}^{3}$ ) with different levels of fines content (3\% or 12\%) were fluidized using ambient air. Experiments were conducted at four different conditions, probing the effects of bed depth (cases 1 and 2) and fines content (cases 3 and 4) on fluidization. Defluidized regions or gas by-passing were observed in cases 1 and 3, which correspond to deep bed and lower fines content, respectively (Shadle et al., 2011). In the present study we consider case 3 for which experimental data were provided in the form of: (a) time-averaged axial gas pressure gradient profile, and (b) mean and standard deviation of differential pressure - across the entire bed as well as across a 0.61-m section at the mid-point elevation of $2.62 \mathrm{~m}$ — at four different equally spaced azimuthal locations.

Although the experiments involved particles with polydisperse size distributions, the present simulations consider only a single particle size, chosen to be the Sauter-mean diameter $(78.66 \mu \mathrm{m})$. The mass-flow rate of gas corresponding to the superficial velocity $(0.6 \mathrm{~m} / \mathrm{s})$ used in the experiments was specified as an inlet boundary condition at the gas distributor. The values of the characteristic quantitieslength, time, stress, and mass flux - used to render all quantities dimensionless are listed in Table 1 of Ozarkar et al. (2015), which also are very comparable to the values listed in Table 1 of the present work. No-slip boundary condition was specified on the wall for both phases. The primary and secondary cyclones in the experiments were replaced by a top exit maintained at atmospheric pressure; the particles escaping through the top outlet were recirculated back into the main section at the secondary cyclone solid return pipe location using a recirculating boundary condition (implemented via a user-defined function in ANSYS Fluent). Gas was allowed to exit from top outlet without special treatment. 
The total mass of particles in the fluidized bed was not provided in the challenge problem statement. Simulations were first done with an initial estimate of $1752 \mathrm{~kg}$ for solids inventory $(2.44 \mathrm{~m}$ static bed height above the distributor with voidage of 0.4 ). The simulations were then repeated for a slightly larger inventory of $1902 \mathrm{~kg}$ to achieve a better match, as discussed below. The grid topology and resolution are also exactly the same as those chosen finally by Ozarkar et al. (2015): 50,000 hybrid grids with three boundary layer grid elements. Simulations were performed with first-order temporal discretization, second-order spatial discretization, and $1 \times 10^{-3}$ convergence criteria for all variables; time-averaged data were gathered for at least $20 \mathrm{~s}$ at a frequency of $50 \mathrm{~Hz}$ after the simulation reached statistical steady state (quantified by the fluid static pressure at multiple elevations). Ozarkar et al. (2015) have already shown that at this grid resolution, both the microscopic drag model and filtered one-marker models fail badly, and that two-marker drag models are essential to capture the NETL/PSRI data.

Figure 20 shows a snapshot of the particle volume fraction field along a vertical plane; coarse spatiotemporal structures are readily seen. Figure 21 shows the time-averaged axial pressure profile. As indicated previously, initial simulations were performed with an estimate of $1752 \mathrm{~kg}$ for solids inventory. Although the absolute values of the predicted pressure are smaller than experimental measurements, the slopes $d P / d y$ match very well - this suggests that our initial estimate for solids inventory needs to be readjusted. With a revised solids of $1902 \mathrm{~kg}$, the simulation prediction agrees with experimental data remarkably well. Note that this adjustment step may have been avoided if the solids inventory had been provided in the NETL/PSRI data set.

The standard deviation of the differential pressure across entire bed and that across a $0.61-\mathrm{m}$ section are used as metrics of fluidization quality (Fig. 22). These standard-deviation measurements are performed, in experiments and simulations, at four azimuthal locations; these four points are equally spaced along the circumferential direction at $90^{\circ}$ intervals (refer to Ozarkar et al. (2015)). The high levels of standard deviation of differential pressure across a $0.61 \mathrm{~m}$ section seen at azimuthal locations 3 and 4 in experiments have been attributed to gas bypassing; the simulations do not reveal this feature. Thus, the 
simulations capture the time-averaged results well, but not some of the dynamic features observed in the experiments. We hypothesize that this deficiency may be attributed to two factors not considered in the simulations: (a) a frictional stress, which is essential to capture defluidized regions, and (b) a more accurate representation of the gas inlet which, in reality, is inhomogeneous across the inflow. At most of the azimuthal locations presented in Fig. 22, the underprediction of the standard deviation of the differential pressure could also be due to the damping introduced by the filtering process which filters out the fine scale structures. Therefore, the inability to precisely match the pressure fluctuations could be an inherent outcome of the filtering process. These factors will be explored in future. Notwithstanding, the predictions shown in Fig. 22 from the current set of models are significantly better compared to the corresponding values predicted using the Milioli et al. (2013) model, as presented in Fig. 7 of Ozarkar et al. (2015). Further discussion of the simulation results and a comparison of predictions provided by different filtered models will be presented in a separate study.

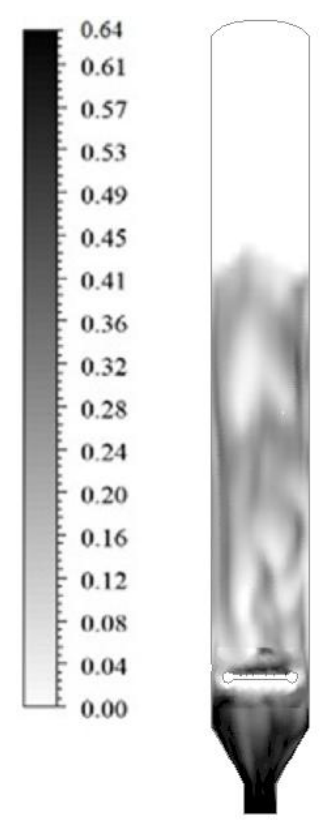

Fig. 20. Instantaneous snapshot of particle volume fraction field inside the test unit extracted at $50 \mathrm{~s}$ of flow time from simulation using the filtered-model closures presented in Table 2. This test-unit design and reference experimental data is provided by the NETL/PSRI challenge problem (Shadle et al., 2011). 


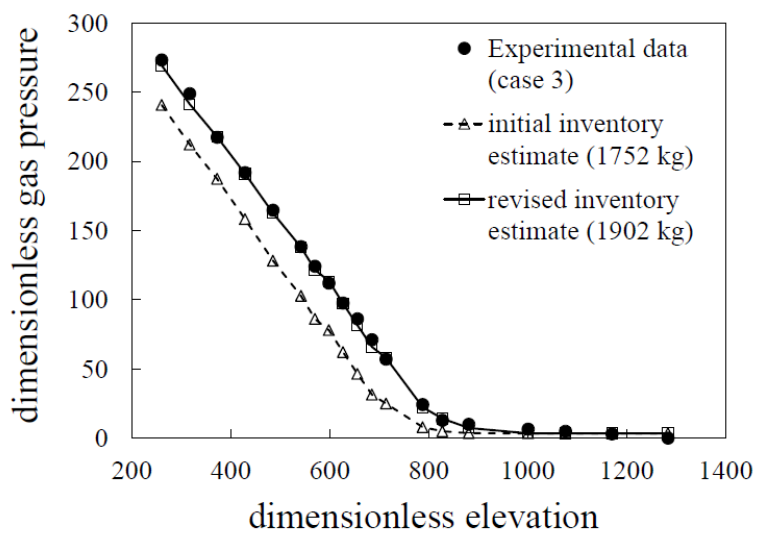

Fig. 21. Dimensionless time-averaged gas pressure profiles plotted along the height of the test unit. Numerical predictions are obtained from simulations of filtered-model closures presented in Table 2, with an initial and subsequently revised estimates of the total solids inventory. The predictions are compared with the NETL-PSRI experimental data (case 3 from Shadle et al., 2011). Gas pressure and elevation are made dimensionless by characteristic stress and length scales, respectively (refer to Table 1).

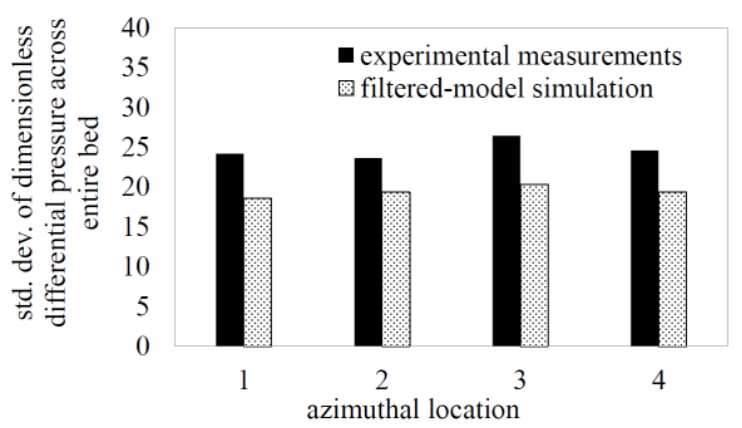

(a)

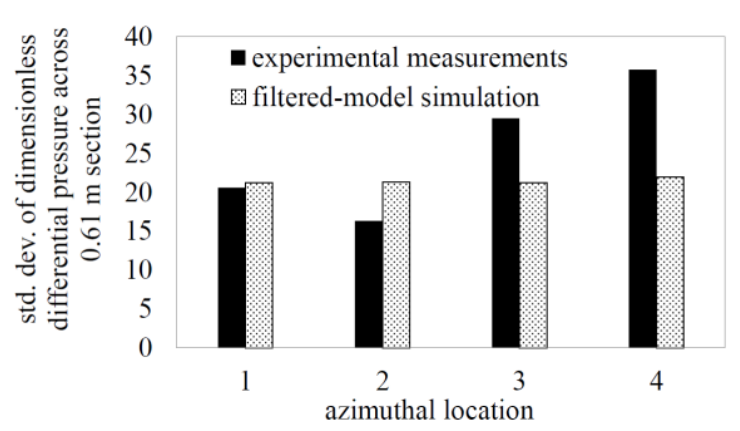

(b)

Fig. 22. Comparison of standard deviations of dimensionless differential pressure across (a) the entire bed and (b) across a 0.61-m section with mid-point elevation of $2.62 \mathrm{~m}$, obtained from simulations of case 3 (see Shadle et al. (2011)). Experimental measurements are compared against predictions using the present filtered-model closure (Table 2) at four azimuthal locations, equally spaced along the circumferential direction (refer to Ozarkar et al. (2015)).

\section{Summary}

New filtered constitutive closures are deduced for gas-particle fluidized beds from highly-resolved 3D simulations. These new filtered relationships are intended for implementation as sub-grid corrections in 
coarse-grid CFD models of commercial-scale devices. Coarse-grid filtered-model simulations are expected to yield macroscopic predictions comparable to fully resolved simulations, but at a fraction of the computational cost.

The closure relations developed in this study were implemented in ANSYS Fluent and used to simulate the NETL/PSRI bubbling-fluidized-bed challenge problem. Comparison of the time-averaged axial pressure profile predicted by coarse-grid simulations with experimental data shows good agreement. The pressure fluctuations captured by the simulations are comparable in magnitude to the experimental results, but the simulations are still unable to robustly reproduce the persistent jet-streaming (gas bypassing) instability observed in the experiments.

Comparisons of results using two different types of particles show that the influence of material properties should also appear in the closure for filtered drag coefficient, but the data generated in this work is insufficient to establish this dependence; this research question merits a separate study. At present, the filtered drag coefficient is captured using two markers: filtered particle volume fraction and slip velocity. Whether or not there exists a better, more fundamental choice for the second marker, instead of slip velocity, remains an open question.

\section{Acknowledgments}

This work, performed at the Pacific Northwest National Laboratory, was funded by the U.S. Department of Energy, Office of Fossil Energy's Carbon Capture Simulation Initiative (CCSI) through the National Energy Technology Laboratory. Pacific Northwest National Laboratory is operated by Battelle Memorial Institute for the U.S. Department of Energy under Contract No. DE-AC05-76RL01830. We

also express our gratitude to Christian C. Milioli (currently at Princeton University), Jean-François Dietiker (NETL), and Janine Carney (NETL) for their assistance with this work. 


\section{Disclaimer}

This report was prepared as an account of work sponsored by an agency of the United States Government. Neither the United States Government nor any agency thereof, nor any of their employees, makes any warranty, express or implied, or assumes any legal liability or responsibility for the accuracy, completeness, or usefulness of any information, apparatus, product, or process disclosed, or represents that its use would not infringe privately owned rights. Reference herein to any specific commercial product, process, or service by trade name, trademark, manufacturer, or otherwise does not necessarily constitute or imply its endorsement, recommendation, or favoring by the United States Government or any agency thereof. The views and opinions of authors expressed herein do not necessarily state or reflect those of the United States Government or any agency thereof.

\section{References}

Agrawal, K., Loezos, P.N., Syamlal, M., Sundaresan, S., 2001. The role of meso-scale structures in rapid gas-solid flows. Journal of Fluid Mechanics 445, 151-185.

Andrews, A.T., Loezos, P.N., Sundaresan, S., 2005. Coarse-grid simulation of gas-particle flows in vertical risers. Industrial \& Engineering Chemistry Research 44, 6022-6037.

Campbell, C.S., 1997. Self-diffusion in granular shear flows. Journal of Fluid Mechanics 348, 85-101.

Ding, J., Gidaspow, D., 1990. A bubbling fluidization model using kinetic theory of granular flow. AIChE Journal 36, 523-538.

Fox, R.O., 2014. On multiphase turbulence models for collisional fluid-particle flows. Journal of Fluid Mechanics 742, 368-424.

Gidaspow, D., 1994. Multiphase flow and fluidization: continuum and kinetic theory descriptions. Academic Press, San Diego.

Glasser, B., Kevrekidis, I., Sundaresan, S., 1996. One-and two-dimensional travelling wave solutions in gas-fluidized beds. Journal of Fluid Mechanics 306, 183-221.

Glasser, B., Kevrekidis, I., Sundaresan, S., 1997. Fully developed travelling wave solutions and bubble formation in fluidized beds. Journal of Fluid Mechanics 334, 157-188.

Glasser, B., Sundaresan, S., Kevrekidis, I., 1998. From bubbles to clusters in fluidized beds. Physical Review Letters 81, 1849. 
Holland, D., Müller, C., Dennis, J., Gladden, L., Sederman, A., 2008. Spatially resolved measurement of anisotropic granular temperature in gas-fluidized beds. Powder Technology 182, 171-181.

Holloway, W., Sun, J., Sundaresan, S., 2012. Effect of microstructural anisotropy on the fluid-particle drag force and the stability of the uniformly fluidized state. Journal of Fluid Mechanics 713, 27-49.

Igci, Y., 2011. Closures for coarse-grid simulation of fluidized gas-particle flows, Chemical and Biological Engineering. Princeton University.

Igci, Y., Andrews, A.T., Sundaresan, S., Pannala, S., O'Brien, T., 2008. Filtered two-fluid models for fluidized gas-particle suspensions. AIChE Journal 54, 1431-1448.

Igci, Y., Pannala, S., Benyahia, S., Sundaresan, S., 2012. Validation Studies on Filtered Model Equations for Gas-Particle Flows in Risers. Industrial \& Engineering Chemistry Research 51, 2094-2103.

Igci, Y., Sundaresan, S., 2011a. Constitutive Models for Filtered Two-Fluid Models of Fluidized GasParticle Flows. Industrial \& Engineering Chemistry Research 50, 13190-13201.

Igci, Y., Sundaresan, S., 2011b. Verification of filtered two-fluid models for gas-particle flows in risers. AIChE Journal 57, 2691-2707.

Li, J., Kwauk, M., 1994. Particle-fluid two-phase flow: the energy-minimization multi-scale method. Metallurgical Industry Press, Beijing, PR China.

Milioli, C.C., Milioli, F.E., Holloway, W., Agrawal, K., Sundaresan, S., 2013. Filtered two-fluid models of fluidized gas-particle flows: New constitutive relations. AIChE Journal 59, 3265-3275.

Miller, D.C., Syamlal, M., Mebane, D.S., Storlie, C., Bhattacharyya, D., Sahinidis, N.V., Agarwal, D., Tong, C., Zitney, S.E., Sarkar, A., Sun, X., Sundaresan, S., Ryan, E., Engel, D., Dale, C., 2014. Carbon Capture Simulation Initiative: a case study in multiscale modeling and new challenges. Annu Rev Chem Biomol Eng 5, 301-323.

Natarajan, V., Hunt, M., Taylor, E., 1995. Local measurements of velocity fluctuations and diffusion coefficients for a granular material flow. Journal of Fluid Mechanics 304, 1-25.

O'Brien, T.J., 2014. A multiphase turbulence theory for gas-solid flows: I. Continuity and momentum equations with Favre-averaging. Powder Technology 265, 83-87.

Ozarkar, S.S., Yan, X., Wang, S., Milioli, C.C., Milioli, F.E., Sundaresan, S., 2015. Validation of filtered two-fluid models for gas-particle flows against experimental data from bubbling fluidized bed. Powder Technology 284, 159-169.

Özel, A., Fede, P., Simonin, O., 2013. Effect of unresolved structures on the Euler-Euler simulation of 3D periodic circulating fluidized of binary mixture, 8th International Conference on Multiphase Flow, Jeju, Korea.

Parmentier, J.-F., Simonin, O., Delsart, O., 2012. A functional subgrid drift velocity model for filtered drag prediction in dense fluidized bed. AIChE Journal 58, 1084-1098.

Pope, S.B., 2000. Turbulent flows, 1st ed. Cambridge university press, Cambridge. 
Sarkar, A., Pan, W., Suh, D., Huckaby, E.D., Sun, X., 2014a. Multiphase flow simulations of a moving fluidized bed regenerator in a carbon capture unit. Powder Technology 265, 35-46.

Sarkar, A., Sun, X., Sundaresan, S., 2013. Sub-grid drag models for horizontal cylinder arrays immersed in gas-particle multiphase flows. Chemical Engineering Science 104, 399-412.

Sarkar, A., Sun, X., Sundaresan, S., 2014b. Verification of sub-grid filtered drag models for gas-particle fluidized beds with immersed cylinder arrays. Chemical Engineering Science 114, 144-154.

Schneiderbauer, S., Pirker, S., 2014. Filtered and heterogeneity-based subgrid modifications for gas-solid drag and solid stresses in bubbling fluidized beds. AIChE Journal 60, 839-854.

Shadle, L., Shahnam, M., Cocco, R., Issangya, A., Guenther, C., Syamlal, M., Spenik, J., Ludlow, J.C., Shaffer, F., Panday, R., Gopalan, B., Dastane, R., 2011. Challenge Problem III, Circulating Fluid Bed X Conference, Sun River Valley, Oregon, USA.

Sundaresan, S., 2000. Modeling the hydrodynamics of multiphase flow reactors: current status and challenges. AIChE Journal 46, 1102-1105.

Wen, Yu, 1966. A Generalized Method for Predicting the Minimum Fluidization Velocity. AIChE Journal 12, 610-612. 


\section{Table 1}

Simulation parameters and characteristic scales used for non-dimensionalization. Values are presented for carbon-capture (CC) and fluid-catalytic-cracking (FCC) particles.

\begin{tabular}{llcc}
\hline \multicolumn{1}{c}{ Property } & Symbol & $\begin{array}{c}\text { Value for CC } \\
\text { particle } \\
\text { simulations }\end{array}$ & $\begin{array}{c}\text { Value for FCC } \\
\text { particle } \\
\text { simulations }\end{array}$ \\
\hline Acceleration due to gravity & $g$ & \multicolumn{2}{c}{$9.81 \mathrm{~m} / \mathrm{s}^{2}$} \\
Gas density & $\rho_{g}$ & $1.142 \mathrm{~kg} / \mathrm{m}^{3}$ \\
Gas viscosity & $\mu_{g}$ & $2 \times 10^{-5} \mathrm{Pa.s}$ \\
Particle diameter & $d_{p}$ & $150 \mu \mathrm{m}$ & $75 \mu \mathrm{m}$ \\
Particle density & $\rho_{s}$ & $441 \mathrm{~kg} / \mathrm{m}^{3}$ & $1500 \mathrm{~kg} / \mathrm{m}^{3}$ \\
Particle-particle restitution coefficient & $e_{p p}$ & 0.9 & 0.9 \\
Characteristic velocity: particle terminal & $v_{t}$ & $0.2697 \mathrm{~m} / \mathrm{s}$ & $0.2297 \mathrm{~m} / \mathrm{s}$ \\
velocity & $v_{t}^{2} / g$ & $7.41 \times 10^{-3} \mathrm{~m}$ & $5.38 \times 10^{-3} \mathrm{~m}$ \\
Characteristic length scale & $\rho_{s} v_{t}^{2}$ & $32.08 \mathrm{~N} / \mathrm{m}^{2}$ & $79.18 \mathrm{~N} / \mathrm{m}^{2}$ \\
Characteristic stress scale & $v_{t} / g$ & $0.0275 \mathrm{~s}$ & $0.0234 \mathrm{~s}$ \\
Characteristic time scale & $\rho_{s} v_{t}^{3} / g$ & $0.8818 \mathrm{~Pa} . \mathrm{s}$ & $1.8543 \mathrm{~Pa} . \mathrm{s}$ \\
Characteristic viscosity & $F r_{p}=v_{t}^{2} /\left(g d_{p}\right)$ & 49.43 & 71.74 \\
Particle Froude number & & & \\
\hline
\end{tabular}


Table 2.

Summary of the filtered two-fluid model and constitutive equations

Filtered mass conservation equations

$$
\begin{aligned}
& \frac{\partial}{\partial t}\left(\rho_{s} \bar{\phi}_{s}\right)+\nabla \cdot\left(\rho_{s} \bar{\phi}_{s} \tilde{\boldsymbol{v}}_{\mathrm{s}}\right)=0 \\
& \frac{\partial}{\partial t}\left(\rho_{g} \bar{\phi}_{g}\right)+\nabla \cdot\left(\rho_{g} \bar{\phi}_{g} \tilde{\boldsymbol{v}}_{\mathrm{g}}\right)=0
\end{aligned}
$$

Filtered momentum balance equations

$$
\begin{aligned}
& \frac{\partial}{\partial t}\left(\rho_{s} \bar{\phi}_{s} \tilde{v}_{s}\right)+\nabla \cdot\left(\rho_{s} \bar{\phi}_{s} \tilde{v}_{s} \tilde{v}_{s}=-\nabla \cdot \bar{\Sigma}_{\mathrm{s}}-\bar{\phi}_{s} \nabla \cdot \bar{\sigma}_{\mathrm{g}}+\left[\overline{\mathbf{f}_{\mathrm{gs}}}-\overline{\phi_{s}^{\prime} \nabla \cdot \sigma_{\mathrm{g}}^{\prime}}\right]+\rho_{s} \bar{\phi}_{s} \mathbf{g}\right. \\
& \frac{\partial}{\partial t}\left(\rho_{\mathrm{g}} \bar{\phi}_{\mathrm{g}} \tilde{\boldsymbol{v}}_{\mathrm{g}}\right)+\nabla \cdot\left(\rho_{\mathrm{g}} \bar{\phi}_{\mathrm{g}} \tilde{\boldsymbol{v}}_{\mathrm{g}} \tilde{\boldsymbol{v}}_{\mathrm{g}}\right)=-\bar{\phi}_{\mathrm{g}} \nabla \cdot \bar{\sigma}_{\mathrm{g}}-\nabla \cdot\left[\rho_{\mathrm{g}} \overline{\phi_{\mathrm{g}} \boldsymbol{v}_{\mathrm{g}}^{\prime} \boldsymbol{v}_{\mathrm{g}}^{\prime}}\right]-\left[\overline{\mathbf{f}_{\mathrm{gs}}}-\overline{\phi_{s} \nabla \cdot \sigma_{\mathrm{g}}^{\prime}}\right]+\rho_{\mathrm{g}} \bar{\phi}_{\mathrm{g}} \mathbf{g}
\end{aligned}
$$

where

$$
\begin{aligned}
& \bar{\Sigma}_{s}=\left[\bar{p}_{s}-\overline{\eta \mu_{b}\left(\nabla \cdot v_{s}\right)}\right] \boldsymbol{I}+\left[\frac{1}{3} \operatorname{tr}\left(\rho_{s} \overline{\phi_{s} \boldsymbol{v}_{s}^{\prime} \boldsymbol{v}_{s}^{\prime}}\right)\right] \boldsymbol{I}-2 \bar{\mu}_{s} \overline{\boldsymbol{s}}_{s}-2\left[\frac{\mid \rho_{s} \phi_{s} v_{s}^{\prime} \boldsymbol{v}_{s}^{\prime}}{\bar{s}_{s, i \neq j}}\right] \overline{\boldsymbol{s}}_{s}+\bar{\sigma}_{s, \text { aniso }} \\
& =\left[\bar{p}_{k i n, s}+\bar{p}_{\text {meso }, s}\right] \boldsymbol{I}-2\left[\bar{\mu}_{s}+\bar{\mu}_{\text {mess }, s}\right] \overline{\boldsymbol{s}}_{s}+\bar{\sigma}_{s, \text { aniso }} \\
& {\left[\rho_{g} \overline{\phi_{g} v_{\mathrm{g}}^{\prime} v_{\mathrm{g}}^{\prime}}\right]=\bar{p}_{\text {meso,g }} \boldsymbol{I}-2 \bar{\mu}_{\text {meso }, g} \bar{s}_{g}} \\
& \overline{\boldsymbol{s}}_{i}=\frac{1}{2}\left[\nabla \tilde{\boldsymbol{v}}_{i}+\left(\nabla \tilde{\boldsymbol{v}}_{i}\right)^{\mathrm{T}}\right]-\frac{1}{3}\left(\nabla \cdot \tilde{\boldsymbol{v}}_{i}\right) \boldsymbol{I} \quad(i=s, g)
\end{aligned}
$$

\section{$\underline{\text { Two-marker gas-particle drag model }}$}

$\overline{\boldsymbol{F}}_{g s}=\left[\overline{\boldsymbol{f}}_{g s}-\overline{\phi_{s}^{\top} \nabla \cdot \sigma_{g}^{\prime}}\right]=\left(1-H_{3 D}^{t w o}\right) \beta_{g s, \text { micro }}\left(\tilde{\boldsymbol{v}}_{g}-\tilde{\boldsymbol{v}}_{s}\right)$

where

$$
H_{3 D}^{t w o}=\min \left[\left(a+\frac{b}{\left|\tilde{\boldsymbol{v}}_{\text {slip }}^{*}\right|}\right) \bar{\phi}_{s}^{\left({ }^{c+} \frac{d}{\tilde{\hat{r}}_{\text {sip }}^{*} \mid}\right)}, 0.97\right]
$$


Table 2. (continued)

$$
\begin{array}{llll}
\Delta_{\text {filter }}^{*}=0.6748 & \Delta_{\text {filter }}^{*}=\text { from } 1.3495 & \text { to } 6.7476 \\
a=0.9240 & c=-0.03102 & a=0.9506 & c=0.049\left(\frac{1}{\Delta_{\text {filter }}^{*}}-1\right) \\
b=0.2370 & d=0.4189 & b=0.1708 & d=0.3358
\end{array}
$$

\section{Filtered particle phase stress}

Filtered particle phase micro-scale pressure:

$$
C_{\text {press }, s}=\frac{\bar{p}_{k i n, s}}{\rho_{s}\left(\Delta_{\text {filter }}\right)^{2} \bar{S}_{s}^{2}} \approx \frac{0.01797\left(\bar{\phi}_{s}\right)^{1.645}}{\bar{\phi}_{s, \max }-\bar{\phi}_{s}} ; \quad \bar{\phi}_{s, \max }=0.65
$$

Filtered particle phase micro-scale viscosity:

$$
C_{\text {visc }, s}=\frac{\bar{\mu}_{s}}{\rho_{s}\left(\frac{g}{v_{t}^{2}}\right)^{-6 / 7}\left(\Delta_{\text {filter }}\right)^{8 / 7} \bar{S}_{s}} \approx \frac{0.00307\left(\bar{\phi}_{s}\right)^{1.544}}{\bar{\phi}_{s, \max }-\bar{\phi}_{s}}
$$

Filtered particle phase meso-scale pressure:

$$
C_{\text {press }, \text { meso }, s}=\frac{\bar{p}_{\text {meso }, s}}{\rho_{s}\left(\frac{g}{v_{t}^{2}}\right)^{3 / 7}\left(\Delta_{\text {filter }}\right)^{17 / 7} \bar{S}_{s}^{2}} \approx \frac{0.0236\left(\bar{\phi}_{s}\right)^{1.115}}{\bar{\phi}_{s, \max }-\bar{\phi}_{s}}
$$

Filtered particle phase meso-scale viscosity:

$$
C_{\text {visc }, \text { meso }, s}=\frac{\bar{\mu}_{\text {meso }, s}}{\rho_{s}\left(\frac{g}{v_{t}^{2}}\right)^{-2 / 7}\left(\Delta_{\text {filter }}\right)^{12 / 7} \bar{S}_{s}} \approx \frac{0.02518\left(\bar{\phi}_{s}\right)^{1.123}}{\bar{\phi}_{s, \max }-\bar{\phi}_{s}}
$$

Filtered particle phase normal stress anisotropy:

$$
\bar{\sigma}_{s, \text { aniso }} \approx\left(\rho_{s} v_{t}^{2}\right)\left[\begin{array}{ccc}
-\frac{1}{3} \bar{N}_{s, 1}^{*} & 0 & 0 \\
0 & +\frac{2}{3} \bar{N}_{s, 1}^{*} & 0 \\
0 & 0 & -\frac{1}{3} \bar{N}_{s, 1}^{*}
\end{array}\right]
$$


Table 2. (continued)

where $\quad \bar{N}_{s, 1}^{*}=\left(\bar{\Sigma}_{s, y y}-\bar{\Sigma}_{s, x x}\right) /\left(\rho_{s} v_{t}^{2}\right) \approx 2.6 \bar{p}_{s, \text { meso }}^{*}\left(1-\frac{\bar{\phi}_{s}}{\bar{\phi}_{s, \max }}\right)^{1.2}$

Note: $\quad \bar{N}_{s, 2}^{*}=\left(\bar{\Sigma}_{s, x x}-\bar{\Sigma}_{s, z z}\right) /\left(\rho_{s} v_{t}^{2}\right) \approx 0$

\section{Filtered gas phase stress}

Filtered gas phase meso-scale pressure:

$$
C_{\text {press }, \text { meso }, g}=\frac{\bar{p}_{\text {meso }, g}}{\rho_{g}\left(\frac{g}{v_{t}^{2}}\right)^{5 / 7}\left(\Delta_{\text {filter }}\right)^{19 / 7} \bar{S}_{g}^{2}} \approx 0.0661+0.0164 \bar{\phi}_{s}-0.194\left(\bar{\phi}_{s}\right)^{2}
$$

Filtered gas phase meso-scale viscosity:

$$
C_{\text {visc }, \text { meso }, g}=\frac{\bar{\mu}_{\text {meso }, g}}{\rho_{g}\left(\Delta_{\text {filter }}\right)^{2} \bar{S}_{g}} \approx 0.0330+0.218 \bar{\phi}_{s}-0.485\left(\bar{\phi}_{s}\right)^{2}
$$


54 solids

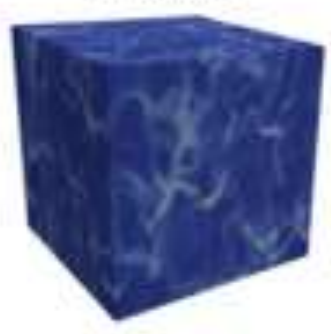

3056 sotids

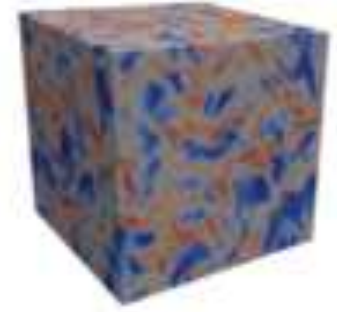

Highly-resolved 3-D simulations

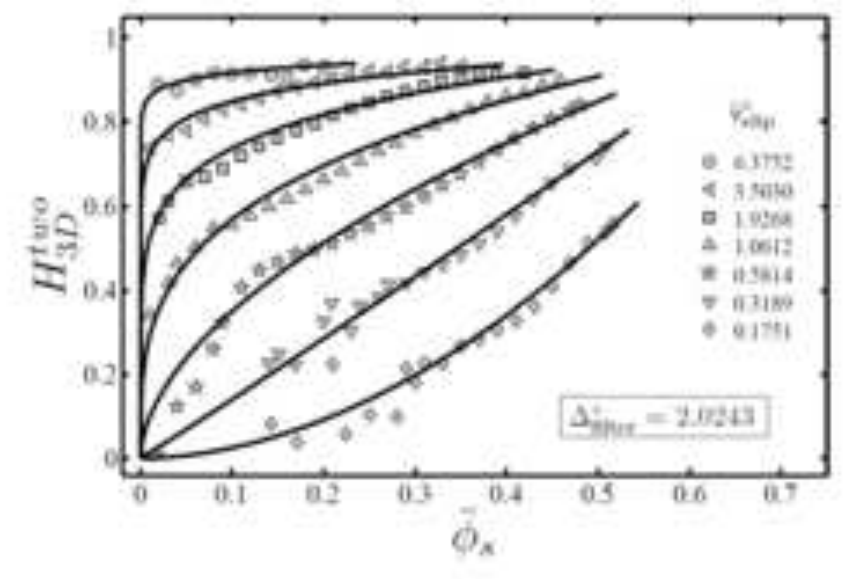

Sub-grid filtered constitutive models

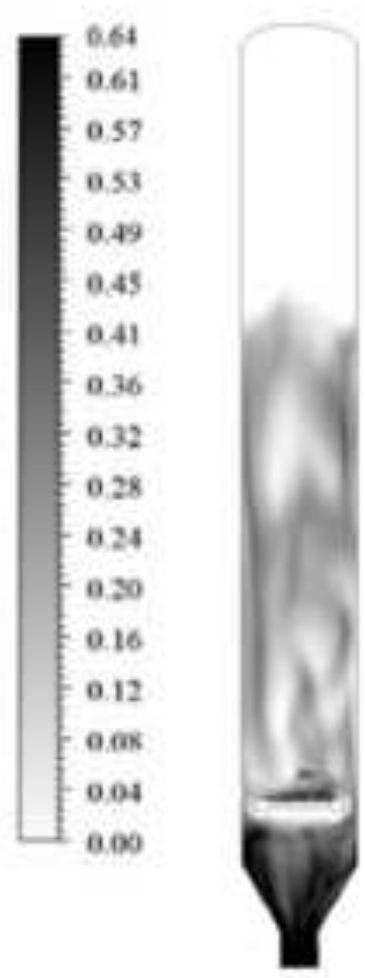

Coarse-grid filtered CFD simulations of large fluidized beds 